UUITP-10/93

FIAN/TD-07/93

ITEP-M4/93 日

April 1993

\title{
Generalized Kazakov-Migdal-Kontsevich Model: group theory aspects
}

\author{
S.Kharchev? A, A.Marshakov?], A.Mironovt \\ Institute for Theoretical Physics, Uppsala University, Box 803, S-751 08 Uppsala, Sweden \\ and \\ Theory Department, P.N.Lebedev Physics Institute, Leninsky prospect, 53, \\ Moscow, 117 924, Russia, \\ A.Morozov占 \\ ITEP , Bol.Cheremushkinskaya, 25, Moscow, 117 259, Russia, \\ and \\ Institute for Theoretical Physics, Uppsala University, Box 803, S-751 08 Uppsala, Sweden
}

\footnotetext{
${ }^{1}$ Preliminary version of this report was circulating as preprint FIAN/TD-01/93, ITEP-M12/92

${ }^{2}$ E-mail address: tdparticle@glas.apc.org, serg@grotte.teorfys.uu.se

${ }^{3}$ E-mail address: tdparticle@glas.apc.org, marshakov@nbivax.nbi.dk

${ }^{4}$ E-mail address: tdparticle@glas.apc.org, mironov@grotte.teorfys.uu.se

${ }^{5}$ E-mail address: tdparticle@glas.apc.org, morozov@vxdesy.desy.de
} 


\begin{abstract}
The Kazakov-Migdal model, if considered as a functional of external fields, can be always represented as an expansion over characters of $G L$ group. The integration over "matter fields" can be interpreted as going over the model (the space of all highest weight representations) of $G L$. In the case of compact unitary groups the integrals should be substituted by discrete sums over weight lattice. The $D=0$ version of the model is the Generalized Kontsevich integral, which in the abovementioned unitary (discrete) situation coincides with partition function of the $2 d$ Yang-Mills theory with the target space of genus $g=0$ and $m=0,1,2$ holes. This particular quantity is always a bilinear combination of characters and appears to be a Toda-lattice $\tau$-function. (This is generalization of the classical statement that individual $G L$ characters are always singular KP $\tau$-functions.) The corresponding element of the Universal Grassmannian is very simple and somewhat similar to the one, arising in investigations of the $c=1$ string models. However, under certain circumstances the formal sum over representations should be evaluated by steepest descent method and this procedure leads to some more complicated elements of Grassmannian. This "Kontsevich phase" as opposed to the simple "character phase" deserves further investigation.
\end{abstract}




\section{Contents}

\begin{tabular}{lll}
\hline 1 & Introduction & 3
\end{tabular}

\begin{tabular}{|l|l|l|l|l}
\hline 2 & IZ formula and characters & 5
\end{tabular}

\begin{tabular}{|lrl}
3 Vertex operators and sewing (topology change) & 8
\end{tabular}

\begin{tabular}{|ll}
\hline Determinant representations of $\tau$-functions & 11
\end{tabular}

5 Characters of GL group as singular KP $\tau$-functions 14

5.1 A notion of singular $\tau$-function $\ldots \ldots \ldots \ldots \ldots \ldots$

5.2 The characters and Shur polynomials $\ldots \ldots \ldots \ldots \ldots$

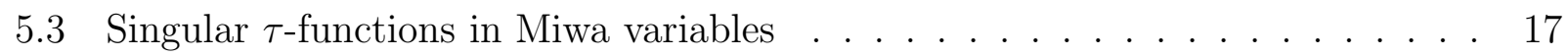

5.4 Character expansion of generic $\tau$-function $\ldots \ldots \ldots . \ldots . \ldots . \ldots$

\begin{tabular}{|ll}
6 & Bilinear combination of characters \\
\hline
\end{tabular}

\begin{tabular}{|lrl}
\hline 7 Comments on Kontsevich model & 26
\end{tabular}

\begin{tabular}{llr}
\hline Concluding remarks & 29
\end{tabular} 


\section{Introduction}

Matrix models remain to be an an important part of modern string theory. Originally developed as an approximate method mostly for the study of Yang-Mills theories, it appeared capable to extract some exact combinatorial information from a wider class of physical models. Restricted class of eigenvalue matrix models provides now the most profound information about topology of module spaces - the underlying structure of (topological) $2 d$ gravity. ) $2 d$ gravity. Further progress of string theory will depend on the possibility to apply the methods, developed for investigation of topological theories to generic string models. Within the field of matrix models the problem is to find adequate generalization of results, obtained for eigenvalue matrix models to the case of generic matrix models, in particular with angular (unitary-matrix) degrees of freedom. The main puzzle is the adequate generalization of integrable structure of matrix models, which plays the crucial role in the eigenvalue model case [1, 2, 3] (see also 4, 5] for a review).

At the boundary between eigenvalue and generic matrix models lies the so called KazakovMigdal model [6], which still can be explicitly represented in terms of eigenvalues, but this representation is already too complicated to be of use by itself. The model has three kinds of matrix variables: Hermitean $N \times N$ matrices $\Phi^{(\alpha)}$ and $L^{(\mu)}$ and unitary $N \times N$ matrices $U^{(\alpha \beta)}, U^{(\alpha \mu)}$, and partition function is

$$
\begin{aligned}
& \mathcal{Z}_{V}\{L\}=\mathcal{C}_{V}\{L\} \int d \Phi^{(\alpha)}\left[d U^{(\alpha \beta)}\right]\left[d U^{(\alpha \mu)}\right] \exp \operatorname{tr}\left(\sum V_{\alpha}\left(\Phi^{(\alpha)}\right)+\right. \\
& \left.+\sum c_{\alpha \beta} \Phi^{(\alpha)} U^{(\alpha \beta)} \Phi^{(\beta)} U^{(\alpha \beta)^{\dagger}}+\sum c_{\alpha \mu} \Phi^{(\alpha)} U^{(\alpha \mu)} L^{(\mu)} U^{(\alpha \mu)^{\dagger}}\right)
\end{aligned}
$$

In the original Kazakov-Migdal model $\alpha$ labels the points of regular $D$-dimensional lattice, and $c_{\alpha \beta} \neq 0$ only for the closest neighbours on the lattice. Also usually all the potentials $V_{\alpha}$ and non-vanishing link couplings $c_{\alpha \beta}$ are the same, and background fields $L^{(\mu)}$ are not introduced. A very special case of the model (11) is the Generalized Kontsevich Model [3] with partition function

$$
\mathcal{Z}_{V}\{L\}=\mathcal{C}_{V}\{L\} \int d \Phi e^{-\operatorname{tr} V(\Phi)+\operatorname{tr} \Phi U L U^{\dagger}}
$$

Below, we will use $G(K M)^{2}$ to denote the "generalized Kazakov-Migdal-Kontsevich Model" (11). 
The crucial feature of $G(K M)^{2}$, which distinguishes it from the naive multimatrix model

$$
\begin{gathered}
\mathcal{Z}_{V}^{m m}\{L\}=\mathcal{C}_{V}\{L\} \int d \Phi^{(\alpha)} \exp \operatorname{tr}\left(\sum V_{\alpha}\left(\Phi^{(\alpha)}\right)+\right. \\
\left.+\sum c_{\alpha \beta} \Phi^{(\alpha)} \Phi^{(\beta)}+\sum c_{\alpha \mu} \Phi^{(\alpha)} L^{(\mu)}\right)
\end{gathered}
$$

is the possibility to represent it as an eigenvalue model, i.e. in the form of integral over eigenvalues $\phi_{i}^{(\alpha)}, i=1 \ldots N$, of matrices $\Phi^{(\alpha)}$. In the case of the naive multimatrix model it is only possible unless there are no closed loops in "target" space. The central ingredient of this transition is the Harish-Chandra-Itzykson-Zuber formula [7]

$$
I\{X, L\}=\int[d U]_{n \times n} e^{\operatorname{tr} X U L U^{\dagger}}=\frac{(2 \pi)^{\frac{n(n-1)}{2}}}{n !} \frac{\operatorname{det}_{i j} e^{l_{i} x_{j}}}{\Delta(L) \Delta(X)}
$$

Making use of this formula one can integrate over link angle variables and rewrite (1) as:

$$
\begin{gathered}
\mathcal{Z}_{V}\{L\}=\mathcal{C}_{V}\{L\} \int \prod d \phi_{i}^{(\alpha)} \times \\
\times \exp \left(\sum V_{\alpha}\left(\phi_{i}^{(\alpha)}\right) \prod I\left\{\Phi^{(\alpha)}, \Phi^{(\beta)} ; c_{\alpha \beta}\right\} \prod I\left\{\Phi^{(\alpha)}, L^{(\mu)} ; c_{\alpha \mu}\right\}\right.
\end{gathered}
$$

This is, however, not a very illuminating expression and some extra ideas are required to investigate its mathematical structure.

One of such ideas is to make use of the group theory structure, implicit in formula (11) and in the entire model (1). In its turn, this kind of structure should be related to integrability. We describe below only some very simple results in this direction, concerning some oversimplified versions of $G(K M)^{2}$, closely related to the model (2). Actually they are not very easy to generalize, but this certainly deserves further investigation. The main motivation is that Generalized Kontsevich model (2) is not by itself sufficient to exhaustively describe even generic $(p, q)$-string models with $d \leq 2(c \leq 1)[\mathbb{6}, 9]$ : only models of the $(p, 1)$-type (and their topological deformations) are represented well enough in this form [10, 11]. The necessary generalizations (related to "multi-component" integable hierarchies) seem to require many background fiels $L$ instead of a single one in (2), and these can be introduced in the manner suggested by eq.(11) without spoiling the solvability of the model. The search of a reasonable generalization of GKM in the framework of $G(K M)^{2}$ is an interesting problem for future investigation. The subject is also closely related to the popular $2 d$ Yang-Mills theory [12][18. 
The group-theoretical description of $G(K M)^{2}$ appears to be somewhat "orthogonal" to the usual presentation of GKM in existing literature. In fact the models possess two different limits to be refered to as the "character" and "Kontsevich" phases. The proper time-variables are different in two limits: they are expanded in positive and negative powers of external fields $L$ in the character and Kontsevich phases respectively, so that the relation between them is a kind of duality ("modular") transformation $L \longrightarrow 1 / L(\log G \longrightarrow 1 / \log G)$. Remarkably, integrability occurs in both phases, but the detailed structure is somewhat different: while it is KP pattern, that arises in the simplest examples of Kontsevich phase, in the character phase it is substituted by a less specific Toda structure, which includes non-trivial dependence on the zero-time (i.e. on the size of the matrix in the matrix-model language). This paper deals mostly with description of the character phase, which, though much simpler, did not yet attract enough attention in the theory of GKM. It is in this phase that generalization of GKM to $G(K M)^{2}$ looks most straightforward.

\section{IZ formula and characters}

The proper derivation of eq.(四) should be on the base of the DH theorem [19] (see [20] for more comments on this approach). Technically simpler is the derivation, proposed in ref. 221, which is based on the equations

$$
\begin{aligned}
& \operatorname{tr}\left(\frac{\partial}{\partial L_{t r}}\right)^{n} I\{X, L\}=\left(\operatorname{tr} X^{n}\right) I\{X, L\} \\
& \operatorname{tr}\left(\frac{\partial}{\partial X_{t r}}\right)^{n} I\{X, L\}=\left(\operatorname{tr} L^{n}\right) I\{X, L\}
\end{aligned}
$$

together with the statement that $I\{X, L\}$ depends only on the eigenvalues of $X$ and $L$. One can easily recognize in these equations the standard differential equations for characters of $G L$ group:

$$
\Delta_{n}(G) \chi_{R}(G)=C_{n}(R) \chi_{R}(G)
$$

where $\chi_{R}(G)=\operatorname{Tr}_{R} G$ is the trace of group element $G$ in representation $R$ and $\Delta_{n}$ is invariant differential operator of the $n$-th order on group (for example, $\Delta_{2}$ is the Laplace operator on group). Indeed,

$$
I\{X, L\} \equiv \frac{\hat{\chi}_{R}(G)}{d_{R}} \sim \frac{\chi_{R}(G)}{d_{R}}
$$


where $G=e^{L}$ and $X$ labels representation $R$ of dimension $d_{R}$, while coefficient of proportionality between $\hat{\chi}_{R}$ and $\chi_{R}$ is the ratio of Van-der-Monde operators $\frac{\Delta(G)}{\Delta(L)}=\frac{\Delta\left(e^{L}\right)}{\Delta(L)}=\prod_{i>j} \frac{e^{l_{i}-e^{l_{j}}}}{l_{i}-l_{j}}$.

In order to get rid of this ratio one can use a more complicated matrix model (quantum mechanics on the coadjoint orbit) [22]:

$$
\frac{\chi_{R}\left(e^{L}\right)}{d_{R}} \equiv \int[D U(t)] \exp \int d t \operatorname{tr} X\left(U^{-1}(t) \partial_{t} U(t)+U^{-1}(t) L U(t)\right)
$$

Perhaps, this 1-dimensional functional integral should be substituted instead of

$$
\frac{\hat{\chi}_{R}\left(e^{L}\right)}{d_{R}}=\int[d U] \exp \operatorname{tr} X U^{-1} L U^{\dagger}
$$

into eq.(四) in order to describe couplings to background fieldsf?

Eqs.(8) and (4) together are nothing but the well-known Weyl character formula. Indeed, each irreducible representation $R$ of $\mathrm{GL}(\mathrm{N})$ is uniquely determined by the set of non-increasing positive numbers ("signature")

$$
R=\left(m_{1}, m_{2}, \ldots m_{N}\right), \quad m_{1} \geq m_{2} \geq \ldots \geq m_{N}
$$

where $m_{i}$ are eigenvalues of the highest vector:

$$
E_{i i} v=m_{i} v, \quad E_{i j} v=0, i<j .
$$

where $E_{i j}$ are representations of the GL(N) basis $e_{i j},\left(e_{i j}\right)_{\alpha \beta}=\delta_{i \alpha} \delta_{j \beta}$ and

$$
\left[E_{i j}, E_{k l}\right]=\delta_{k j} E_{i l}-\delta_{i l} E_{k j}
$$

Every irreducible representation can be determined by the only knowledge of the characters. First Weyl formula for (primitive) characters reads:

$$
\chi=\frac{\operatorname{det} \lambda_{j}^{m_{i}+N-j}}{\prod_{i<j}\left(\lambda_{i}-\lambda_{j}\right)}
$$

where $\lambda_{i}$ are eigenvalues of the given matrix $G \in G L(N)$ and $\left\{m_{i}\right\}$ being the lengths of the rows of corresponding Young table.

\footnotetext{
${ }^{1}$ This formula can be considered as one-dimensional Wess-Zumino model. Similarly, the two-dimensional $W Z W$ model gives rise to Kac-Moody characters.

${ }^{2}$ Note that this procedure actually shifts the dimension $D \longrightarrow D+1$.
} 
Equivalently, every irreducible representation can be characterized by numbers

$$
\left(k_{1}, k_{2}, \ldots, k_{N}\right) ; k_{1}>k_{2}>\ldots k_{N} \geq 0
$$

where

$$
k_{i}=m_{i}+N-i
$$

The second Weyl formula for (primitive) characters (we will use also $\Delta(\lambda) \equiv \prod_{i>j}\left(\lambda_{i}-\lambda_{j}\right)$ ) :

$$
\frac{\operatorname{det} \lambda_{j}^{k_{i}}}{\Delta(\lambda)}=\operatorname{det} P_{k_{i}-j+1}(T)
$$

where $P_{k}(T)$ are the Shur polynomials and times $\left\{T_{k}\right\}$ appear in the form of Miwa transformation

$$
T_{k}=\frac{1}{k} \sum_{i=1}^{N} \lambda_{i}^{k}
$$

It is only for integer values of $k_{i}$ 's that characters indeed depend on the compact-group elements $G=e^{L}$ rather than on those of the non-compact algebra. In this case the value of the character at $G=I(L=0)$ is equal to dimension $d_{R}=\chi_{R}(I)$ of the representation $R$,

$$
d_{R}=\Delta(k)=\prod_{i>j} \frac{k_{i}-k_{j}}{i-j} .
$$

Thus we obtain interpretation of the $G(K M)^{2}$ as a theory where integration (sum) at every point $\alpha$ is going over all representations of $G L$ algebra, labeled by eigenvalues of matrices $\Phi_{\alpha}$ (i.e. integral at every point $\alpha$ is over a model space of $G L$ ). This implies a natural modification of the model for the case of unitary group: integrals over eigenvalues of $\Phi_{\alpha}$ should be substituted by discrete sums over module spaces, namely over integer-valued eigenvalues. In the case of the original GKM (2) this trick would give:

$$
\int \Delta(x) \ldots \rightarrow \sum d_{R} \chi_{R}(G) \ldots
$$

If $n$ background fields are attached to the single point (to form a sphere with $n$ holes), we have:

$$
\int(\Delta(x))^{(2-n)} \ldots \rightarrow \sum_{R} d_{R}^{(2-n)} \prod \chi_{R}\left(G_{\mu}\right) \ldots
$$




\section{$3 \quad$ Vertex operators and sewing (topology change)}

One can consider the terms with external fields in (1) as insertions of a kind of "vertex operators"

$$
\mathcal{V}_{L}(X)=\int[d U] e^{\operatorname{tr} L U X U^{\dagger}}
$$

This expression is similar to $e^{i p x}$ in bosonic string model, and $L$ can be considered as a kind of external momentum. Perhaps, this set of operators should be enlarged to include more complicated integrals with $U$-dependent measures, like?

$$
\mathcal{V}_{L}\{F, X\}=\int[d U] F(U) e^{\operatorname{tr} L U X U^{\dagger}}
$$

as well as some integrals depending on $X=\Phi^{(\alpha)}$ at different sites $\alpha$ (i.e. analogues of $h(\partial x) e^{i p x}$; also note that the analogue of $\int e^{i p x(z)} d^{2} z$ for many site $G(K M)^{2}$ is rather $\sum_{\alpha} \mathcal{V}_{L}\left(X^{(\alpha)}\right)$ than $\mathcal{V}_{L}(X)$ itself).

Given an adequate set of vertex operators one can perform sewing procedure, i.e. go from one collection of sites $\alpha$ and links $\left\langle\alpha \beta>\left.\right|_{c_{\alpha \beta} \neq 0}\right.$ to another (topology changing). In the case of (1) one can take two external fields $L_{\mu}$ and $L_{\nu}$, attached to two sites $\alpha$ and $\beta$ and integrate over these $L$ 's with invariant measure $\iint d L_{\mu} d L_{\nu} \delta\left(L_{\mu}+L_{\nu}\right)$. As result the two fields $X_{\alpha}$ and $X_{\beta}$ will be identified and insertion of

$$
\Delta\left(X^{(\alpha)}\right)^{-2} \delta\left(X^{(\alpha)}-X^{(\beta)}\right)
$$

appears in the integrand in (1). Thus with this set of vertex operators we obtain a specific sewing prescription. Still it seems to be of interest, especially because of its relevance to the $2 d$ Yang-Mills theory, where sewing is known to produce a factor of $d_{R}^{-2}$. Sewing procedure can be modified by changing (enlarging) the set of vertex operators (intermediate states) or varying the form of the three-vertex. Note that so defined sewing procedure is not the same as changing $c_{\alpha \beta}$ to ours in the $G(K M)^{2}(1)$.

If vertex operators were introduced by the rule (9) instead of (21),

$$
\hat{V}_{L}(X)=\int[d U(t)] \exp \int d t \operatorname{tr} X\left(U^{-1}(t) \partial_{t} U(t)+U^{-1}(t) L U(t)\right),
$$

\footnotetext{
${ }^{3}$ See [20, 23, 24] for discussion of generalizations of Itzykson-Zuber formula, adequate for calculations with
} such operators. 
one should sew with the help of the measure $\left[d e^{L}\right]$ instead of $d L$. In terms of characters the two relevant orthogonality conditions are:

$$
\begin{gathered}
\int \hat{\chi}_{R_{1}}\left(e^{L}\right) \hat{\chi}_{R_{2}}\left(e^{-L}\right) d L=\delta_{R_{1} R_{2}}, \\
\int \chi_{R_{1}}\left(e^{L}\right) \chi_{R_{2}}\left(e^{-L}\right)\left[d e^{L}\right]=\delta_{R_{1} R_{2}} .
\end{gathered}
$$

One can consider the above described sewing procedure also more formally just as a consistent set of rules. Then, having the propagator (corresponding to a sphere with two holes)

$$
\sum_{\alpha} \chi_{\alpha}\left(U_{1}\right) \chi_{\alpha}\left(U_{2}\right)
$$

and the wave function (corresponding to the sphere with a hole) $\sum_{\alpha} \chi_{\alpha}(U) d_{\alpha}$, as well as the consistent rules of gluing the hole by putting corresponsding $U=1$ and of gluing two holes just by integrating with the proper measure (induced by (25)), one still has a freedom to choose three point function.

$$
\sum_{\alpha, \beta, \gamma} C_{\alpha \beta \gamma} \chi_{\alpha} \chi_{\beta} \chi_{\gamma}
$$

Certainly, there are some consistency requirements like duality. We do not know all possible solutions of these, but two natural choices are (properly normalized) delta-function coefficients and inverse Clebsh-Gordon coefficients. The normalization might be fixed, say, from the requirement

$$
\sum_{\alpha, \beta} C_{\alpha \beta \gamma} d_{\alpha} d_{\beta}=d_{\gamma}
$$

Then, the first case corresponds just to $2 d$ Yang-Mills theory [14, 12, 26], while the second one describes the correlators in $k \rightarrow \infty$ WZW theory [27].

Further point to be mentioned is that the quantities which we discussed appear related to $2 d$ Yang-Mills correlators with fixed monodromies at the boundaries. Og great interest are also correlators of the Wilson loops. These objects are much more complicated, even for the three-point function at genus zero the answer being proportional to a Clebsh-Gordon coefficient. In general case, it is represented in the form of group integrals and explicit answers are not known.

Now let us brieflt discuss "illustrated version" of the sewing considered in the section. $G(K M)^{2}$ as defined by eq.(1) allows a simple pictorial representation. "Feynman diagramms"

\footnotetext{
${ }^{4}$ We are grateful to A.Gorsky and N.Nekrasov for the discussion of these points.
} 


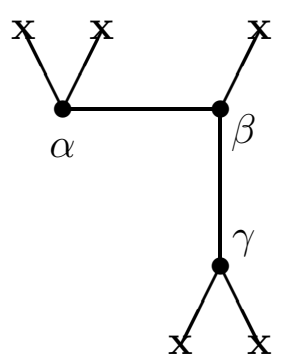

a)

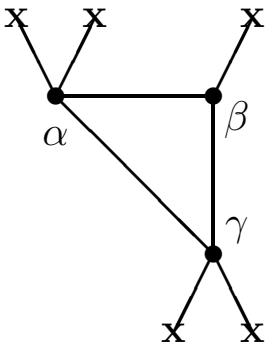

a)

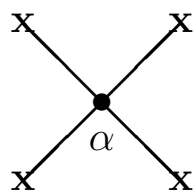

b)

Figure 1

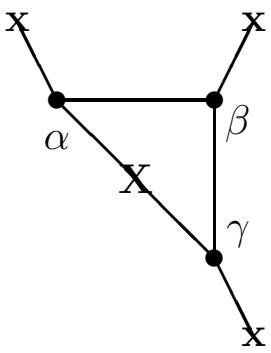

b)

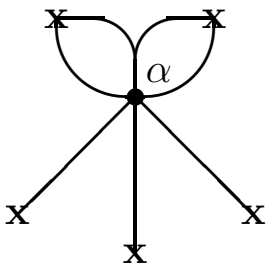

a)

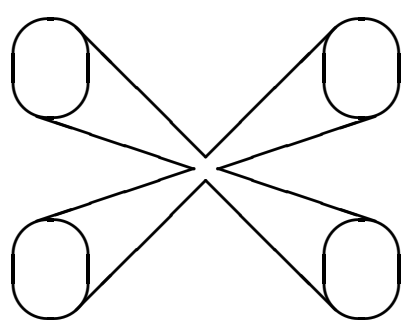

c)

Figure2

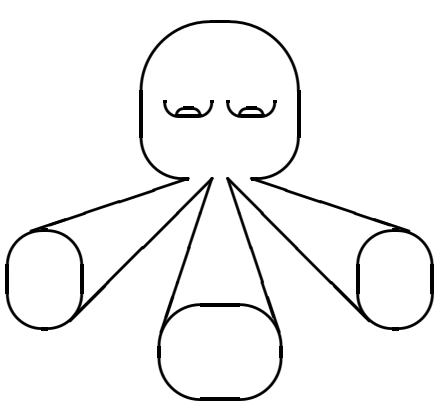

b)

Figure 3

Figure 4

are collections of points and crosses (crosses standing for background fields), connected by links (which depict IZ integrals (田), see Fig.1a.

A link appears between the points $\alpha$ and $\beta$ whenever $c_{\alpha \beta} \neq 0$ and the cross $\mu$ is attached to the point $\alpha$ whenever $c_{\alpha \mu} \neq 0$. The simplest example (which we shall often refer to as $D=0$ model) occurs when there is just a single point, Fig.1b. Conventional GKM arises when the cross is also unique, Fig.1c. If external fields are introduced by the rule (9) instead of (何), links are effectively substituted by tubes, thus a sphere with holes, Fig.2, emerges instead of a hedgehog in Fig.1b. Refleting the difference between points and holes, the relevant vertex operators (24) are, of course, non-local. There are two ways of sewing two points, say, $\alpha$ and $\gamma$ in Fig.1a: either they can be connected by a new link, Fig.3a, or the two attached external fields can be identified and then integrated over according to the rule (23), Fig.3b.

When the latter prescription is used, the $D=0$ model acquires contributions like Fig.4a 
or Fig.4b, depending on the way the external fields are introduced. These pictures makes identification of the $D=0 G(K M)^{2}$ with $2 d$ YM theory rather appealing. They also suggest that appropriate generalization of IZ integrals, which would effectively turn links into tubes, deserves being looked for. The last task is, however, beyond the scope of this paper.

\section{Determinant representations of $\tau$-functions}

In what follows we are going to discuss integrability properties of partition functions, constructed from characters. Therefore we need to remind briefly the main notions from the theory of $\tau$-functions (more formulas which will be used in the next sections are contained in the Appenidices A).

We restrict ourselves to consideration of ordinary KP and Toda-lattice $\tau$-functions, which can be constructed as correlators in the theory of free fermions $\psi, \psi^{*}(b, c$-system of spin 1/2). The basic quantity is the ratio of fermionic correlators,

$$
\tau_{N}(t, \bar{t} \mid \mathcal{G}) \equiv \frac{\left\langle N\left|e^{H} \mathcal{G} e^{\bar{H}}\right| N\right\rangle}{\langle N|\mathcal{G}| N\rangle}
$$

in the theory of free 2-dimensional fermionic fields $\psi(z), \psi^{*}(z)$ with the action $\int \psi^{*} \bar{\partial} \psi$ (see the Appendix A1 for the definitions and notations). In the definition (26)

$$
H=\sum_{k>0} t_{k} J_{k} ; \quad \bar{H}=\sum_{k>0} \bar{t}_{k} J_{-k}
$$

and the currents are defined to be

$$
J(z)=\psi^{*}(z) \psi(z)
$$

where $\psi(z)=\sum_{\mathbf{Z}} \psi_{n} z^{n} d z^{1 / 2}, \psi^{*}(z)=\sum_{\mathbf{Z}} \psi_{n}^{*} z^{-n-1} d z^{1 / 2}$.

This expression for $\tau$-function is rather famous. Let us point out that this $\tau$-function is the $\tau$-function of the most general one-component hierarchy which is Toda lattice hierarchy. Putting all negative times to be zero as well as discrete index $n$, one obtains usual KP hierarchy. An element

$$
\mathcal{G}=: \exp \left\{\sum_{m, n} \mathcal{A}_{m n} \psi_{m}^{*} \psi_{n}\right\}
$$


is an element of the group $G L(\infty)$ realized in the infinite dimensional Grassmannian. The normal ordering should be understood here with respect to any $|k\rangle$ vacuum,f f where vacuum states are defined by conditions

$$
\psi_{m}|k\rangle=0 \quad m<k, \psi_{m}^{*}|k\rangle=0 \quad m \geq k .
$$

After Miwa transformation of time variables,

$$
\begin{gathered}
t_{k}=-\frac{1}{k} \sum_{i=1}^{n}\left(\mu_{i}^{-k}-\lambda_{i}^{-k}\right) ; \\
\bar{t}_{k}=-\frac{1}{k} \sum_{i=1}^{n}\left(\bar{\mu}_{i}^{k}-\bar{\lambda}_{i}^{k}\right),
\end{gathered}
$$

the $\tau$-functions acquires the form of $\square$

$$
\begin{gathered}
\tau_{N}(t, \bar{t} \mid \mathcal{G})=\frac{1}{\Delta(\lambda) \Delta(\mu) \Delta(\bar{\lambda}) \Delta(\bar{\mu})} \times \\
\times \frac{\left\langle N\left|\prod_{i=1}^{n} \psi\left(\lambda_{i}\right) \prod_{i=1}^{n} \psi^{*}\left(\mu_{i}\right) \mathcal{G} \prod_{i=1}^{n} \psi\left(\bar{\mu}_{i}\right) \prod_{i=1}^{n} \psi^{*}\left(\bar{\lambda}_{i}\right)\right| N\right\rangle}{\langle N|\mathcal{G}| N\rangle}
\end{gathered}
$$

Now Wick theorem can be used (the choice of $\mathcal{G}$ as quadratic exponent in fermionic fields is important here) in order to rewrite the r.h.s. in the form of determinant of $2 n \times 2 n$ matrix, with pair correlators as the entries:

$$
\tau_{N}(t, \bar{t} \mid \mathcal{G})=\frac{1}{\Delta(\lambda) \Delta(\mu) \Delta(\bar{\lambda}) \Delta(\bar{\mu})} \operatorname{det}\left(\begin{array}{cc}
\frac{\left\langle N\left|\psi\left(\lambda_{i}\right) \psi^{*}\left(\mu_{i}\right) \mathcal{G}\right| N\right\rangle}{\langle N|\mathcal{G}| N\rangle} & \frac{\left\langle N\left|\psi\left(\lambda_{i}\right) \mathcal{G} \psi^{*}\left(\bar{\lambda}_{i}\right)\right| N\right\rangle}{\langle N|\mathcal{G}| N\rangle} \\
\frac{\left\langle N\left|\psi^{*}\left(\mu_{i}\right) \mathcal{G} \psi\left(\bar{\mu}_{i}\right)\right| N\right\rangle}{\langle N|\mathcal{G}| N\rangle} & \frac{\left\langle N\left|\mathcal{G} \psi^{*}\left(\bar{\lambda}_{i}\right) \psi\left(\bar{\mu}_{i}\right)\right| N\right\rangle}{\langle N|\mathcal{G}| N\rangle}
\end{array}\right)
$$

This expresion can be considerably simplified for special choices of points $\lambda$. There are two especially important particular cases. First, let us put all $\bar{\lambda}$ and $\bar{\mu}$ equal to zero. Then the right lower block of the matrix in (33) is singular and the contributions of the off-diagonal blocks can be neglected. The singularity is canceled by the zeroes, coming from the Van-der-Monde determinants, and we obtain:

\footnotetext{
${ }^{5}$ Indeed, it is rather crucial point and we return to possible choices of the normal orderings in the section 5.

${ }^{6}$ We choose number $n$ of all the four $\lambda$-variables the same. This restriction can be take off eliminating $\lambda$ by puting it equal to $\infty$, while every $\bar{\lambda}$-variable is eliminated by taking it equal to zero.

${ }^{7}$ The simplest way to demonstrate this is to apply the bosonization formulas as in the Appendix A1.
} 


$$
\tau_{N}(t \mid \mathcal{G})=\frac{\prod\left(\mu_{i}-\lambda_{j}\right) \operatorname{det}_{i j} C^{11}\left(\mu_{i}, \lambda_{j}\right)}{\Delta(\lambda) \Delta(\mu)}
$$

with

$$
C^{11}\left(\mu_{i}, \lambda_{j}\right) \equiv \frac{\left\langle N\left|\psi\left(\lambda_{i}\right) \psi^{*}\left(\mu_{i}\right) \mathcal{G}\right| N\right\rangle}{\langle N|\mathcal{G}| N\rangle}
$$

One can further tend all the $\lambda$ to $\infty$ and obtain:

$$
\tau_{N}(t \mid \mathcal{G})=\frac{\operatorname{det}_{i j} \phi_{i}\left(\mu_{j}\right)}{\Delta(\mu)}
$$

with

$$
\phi_{i}(\mu) \equiv \frac{1}{(i-1) !} \lim _{t \rightarrow 0} \partial_{t}^{i-1} \frac{\left\langle N\left|\psi\left(t^{-1}\right) \psi^{*}(\mu) \mathcal{G}\right| N\right\rangle}{\langle N|\mathcal{G}| N\rangle}=\mu^{i-1}\left(1+\mathcal{O}\left(\mu^{-1}\right)\right) .
$$

This is the expression for KP $\tau$-function in Miwa coordinates, discussed in much details in [3.

Another particular case of eq.(33) arises when

$$
\text { all } \lambda \rightarrow \infty, \quad \text { while all } \bar{\lambda} \rightarrow 0
$$

Then (33) turns into not simple at all, and in fact it is better to take the limit (38) directly in the original correlator (32). Then (32) turns into (see the Appendix A2)

$$
\begin{gathered}
\tau_{N}(t, \bar{t} \mid \mathcal{G})=\text { Van-der-Mondes } \times \\
\times \frac{\left\langle N-n\left|\prod_{i=1}^{n} \psi\left(\lambda_{i}\right) \mathcal{G} \prod_{i=1}^{n} \psi^{*}\left(\bar{\lambda}_{i}\right)\right| N-n\right\rangle}{\langle N|\mathcal{G}| N\rangle},
\end{gathered}
$$

and application of Wick theorem provides a determinant of $n \times n$ matrix:

$$
\begin{gathered}
\tau_{N}(t, \bar{t})=\frac{\langle N-n|\mathcal{G}| N-n\rangle}{\Delta\left(z^{-1}\right) \Delta(w)} \times \\
\times \operatorname{det} \frac{\left\langle N-n+1\left|e^{\sum \frac{1}{k} J_{k} z_{i}^{-k}} \mathcal{G} e^{\sum \frac{1}{k} \bar{J}_{k} w_{j}^{k}}\right| N-n+1\right\rangle}{\langle N-n|\mathcal{G}| N-n\rangle}
\end{gathered}
$$

Among all above determinants of correlators, the representation (37) can be immediately interpreted in terms of Baker-Akhiezer (BA) function. Indeed, it can be expressed through the fermionic correlator as follows: 


$$
\Psi_{N}\left(\mu, t_{k}\right) \equiv \frac{\tau\left(t_{k}-\frac{1}{k \mu^{k}}\right)}{\tau\left(t_{k}\right)} e^{\sum t_{k} \mu^{k}}=\frac{\left\langle N+1\left|e^{H} \psi(\mu) \mathcal{G}\right| N\right\rangle}{\left\langle N\left|e^{H} \mathcal{G}\right| N\right\rangle}
$$

The analogous expression is certainly correct for the conjugated function. If we consider Toda lattice case, i.e. allows one to put fermions to the right side with respect to $\mathcal{G}$, we get four different BA functions. Now we easily express the entries of the determinant (37) through one of these functions:

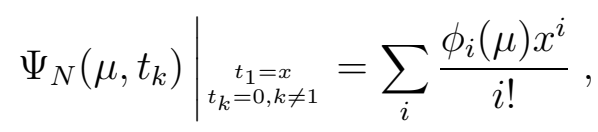

i.e.

$$
\phi_{i}(\mu)=\left.\frac{\partial^{i-1} \Psi_{N}\left(\mu, t_{k}\right)}{\partial x^{i-1}}\right|_{\substack{t_{1}=x \\ t_{k}=0, k \neq 1}} .
$$

As for the Toda lattice case, the entries of the determinant (33) are equal to the BA-functions only asymptotically. Put it differently, BA functions describes the transition amplitudes to the vacuum state, while the determinant (33) contains the transition amplitudes between all one-particle states.

\section{Characters of GL group as singular KP $\tau$-functions}

\subsection{A notion of singular $\tau$-function}

Characters of irreps of GL(N) and SL(N) (see [25]) are given by formulas (13) and (16). Now we are going to demonstrate that these are indeed $\tau$-functions. At first, we consider the $\tau$-function in terms of times. We follow the classical paper [28] and consider for the sake of brevity only KP, not Toda hierarchy.

There is a subspace in the Grassmannian which corresponds to the sigular $\tau$-functions. It means that it can be described in the following equivalent ways:

i) The v.e.v. of the element $\mathcal{G}$ is equal to zero.

ii) It can not be written in the canonical exponential form (29) in some of natural normal orderings. 
iii) It can be immediately related to the characters of group $G L(N)$ at finite $N$.

iv) The $\tau$-function is a polynomial of times (rational solutions to the KP hierarchy).

v) The $\tau$-function can be represented in the determinant form of (36), but no preserving the property of $\phi_{i}(\mu) \sim \mu^{i-1}+O(1 / \mu)$.

Now we are going to comment more on these statements and their interrelations. To begin with, we discuss the case of $i$ ). It implies that the expressions of the type (37) require some regularization which changes the asymptotics of the entries in the determinant and lead to the statement $v$ ). We consider how to do this in the subsection 5.3.

Let us stress that the exponential form of the element of the Grassmannian (29) hints to the non-zero v.e.v. Indeed, one should also take into account the normal ordering. There are two natural normal orderings - these are with respect to the vacuum state $\mid 0>$ and to the empty vacuum state $\mid+\infty>$ (see the Appendices A1 and A3). The first one implies that all annihilation operators to be shifted to the right are $\psi_{k}^{*}$ with $k \geq 0$ and $\psi_{k}$ with $k<0$. The second normal ordering implies all operators with no asterisques standing at the right.

If we represent now the element of the Grassmannian as a normal ordered exponent bilinear in fermions, then the matrices $\mathcal{A}_{m n}$ of the bilinear forms (29) for different normal orderings and for the exponent unordered at all are related in an explicit way [28]. It is obviously that one can not obtain zero v.e.v. from non-singular $\mathcal{A}_{m n}^{\text {unord }}$, as it should be always 1 . The same is correct for the non-singular $\mathcal{A}_{m n}^{1 \text { ord }}$. Thus, one is led to consider the second normal ordering, which is singular in this case. Thus, we have explained the statement ii).

\subsection{The characters and Shur polynomials}

Let us demonstrate the manifest construction of an element of the Grassmannian corresponding to the singular $\tau$-functions. Hereafter, we consider only the second normal ordering. Then, we consider $\mathcal{G}^{(m, n)}=: \exp \left\{\left(\psi_{-m}+\psi_{n}\right)\left(\psi_{-m}^{*}+\psi_{n}^{*}\right)\right\}:(m$ and $n$ are integers) which simplifies when acting on the right vacuum:

\footnotetext{
${ }^{8}$ Note that the first ordering, associated to forced hierarchies [29, 30] which describe matrix models before taking the continuum limit, leads to an infinite matrix $\mathcal{G}_{m n}$ in (29). In the case, the only proper normal ordering of mentioned above is the first one.
} 


$$
\mathcal{G}^{(m, n)}\left|0>=\left[1+\left(\psi_{-m}+\psi_{n}\right)\left(\psi_{-m}^{*}+\psi_{n}^{*}\right)\right]\right| 0>=\psi_{-m}^{*} \psi_{n} \mid 0>.
$$

Now one can trivially calculate the $\tau$-function (see (A25), (A26), (A30) and (A31)):

$$
\begin{gathered}
\tau\left(t_{k}, \mathcal{G}^{(m, n)}\right)=<0\left|e^{H(t)} \psi_{-m}^{*} \psi_{n}\right| 0>= \\
=<0\left|\psi_{-m}^{*}(t) \psi_{n}(t)\right| 0>=\sum_{l \geq 0} P_{l+m}\left(-t_{k}\right) P_{n-l}\left(t_{k}\right),
\end{gathered}
$$

where Shur polynomials $P\left(t_{k}\right)$ are defined

$$
\exp \left\{\sum_{k>0} t_{k} x^{k}\right\} \equiv \sum_{k>0} P_{k}\left(t_{k}\right) x^{k} .
$$

Comparing this result with the second Weyl formula for (primitive) characters, one can see that (5.2) describes the character $\chi_{m, n}$ of the representation corresponding to the "hook" Young diagramm with the row of the length $n+1$ and the column of the length $m$. .

Now it is trivial to generalize the consideration. Indeed, let us consider more complicated element of the Grassmannian $\mathcal{G}^{(\{m\},\{n\})} \equiv: \exp \left\{\prod_{i}\left(\psi_{-m_{i}}+\psi_{n_{i}}\right)\left(\psi_{-m_{i}}^{*}+\psi_{n_{i}}^{*}\right)\right\}$ : which is again equal on the right vacuum to

$$
\mathcal{G}^{(\{m\},\{n\})}\left|0>=\prod_{i} \psi_{-m_{i}}^{*} \psi_{n_{i}}\right| 0>
$$

and the $\tau$-function is

$$
\begin{gathered}
\tau\left(t_{k}, \mathcal{G}^{(\{m\},\{n\})}\right)=<0\left|e^{H(t)} \prod_{i} \psi_{-m_{i}}^{*} \psi_{n_{i}}\right| 0> \\
<0\left|\prod_{i} \psi_{-m_{i}}^{*}(t) \psi_{n_{i}}(t)\right| 0>=\operatorname{det}_{i j}<0\left|\psi_{-m_{i}}^{*}(t) \psi_{n_{j}}(t)\right| 0>=\operatorname{det}_{i j} \chi_{m_{i} n_{j}} .
\end{gathered}
$$

Now, from the textbooks (see, for example, [31]) it is known that if one chooses a sufficiently large integer $N$ and parameterize an element $\mathcal{G}$ of $G L(N)$ as $t_{k} \equiv \frac{1}{k} \operatorname{tr} \mathcal{G}^{k}$, then the character corresponding to the Young diagramm with $l$ "hooks" (i.e. parameterized by $n_{i}, m_{i}, \quad i=$

\footnotetext{
${ }^{9}$ The simplest way to do this is to calculate the matrix of rotations (A24) for the element $\mathcal{G}^{(m, n)}$ and, then, to use formulas ( $\mathrm{A38})$, (A39). It automatically produces the correct determinant form as in formula (16). One can also just use the identities for Shur polynomials obtaining the same result.
} 
$1, \ldots, l)$ is a polynomial in $t_{k}$ independent of the choice of large $N$, T which is given explicitly by formula (14). Again, it is also easy to turn this formula to the second Weyl formula (see the last footnote).

Thus, we have demonstrated the statements iii) and iv) and are going to explain how it is possible using Miwa parameterization, to reproduce in the framework of the present paper the first Weyl formula. Simultaneously we explain the statement $v$ ).

\subsection{Singular $\tau$-functions in Miwa variables}

Thus, we derive the expression for basis vector for the case of the singular $\tau$-functions. To do this, let us consider again in details all the stuff connected with the elementary representation (of $G L(N)$-group) given by hook Young diagramm which contains a row of $n+1$ boxes and a column of $m$ boxes. We already established that the corresponding $\tau$-function is equal to proper determinant with entries linear in Shur polynomials (46) and simultaneously to the following expression:

$$
\chi_{m, n}=\sum_{k=0}^{n} P_{k+m}\left(\left\{-t_{k}\right\}\right) P_{n-k}\left(\left\{t_{k}\right\}\right) .
$$

When $m=1$ this is equal to $P_{n+1}\left(\left\{t_{k}\right\}\right)$ and should be equal to the character of the representation given by the Young diagramm consisting of a line of $n$ boxes. To convince oneself that all listed formulas are correct it is sufficient to write down the manifest expressions for Shur polynomials in Miwa variables (their number will be $N$, therefore all determinants have a size $N \times N)$ :

$$
P_{n}(t)=\frac{(-)^{n}}{\Delta(\mu)} \operatorname{det}\left|\begin{array}{c}
\left\{\mu_{i}^{-n}\right\} \\
\left\{\mu_{i}\right\} \\
\cdots
\end{array}\right|
$$

There is an analogous expression for $P(-t)$ through Miwa variables:

\footnotetext{
${ }^{10}$ Which proves the statement $\left.i v\right)$.
} 


$$
P_{n}(-t)=\frac{(-)^{n}}{\Delta(\mu)} \operatorname{det}\left|\begin{array}{c}
\left\{\mu_{i}^{0}\right\} \\
\left\{\mu_{i}\right\} \\
\ldots \\
\left\{\mu_{i}^{n-1}\right\} \\
\left\{\mu_{i}^{-1}\right\} \\
\left\{\mu_{i}^{n}\right\} \\
\ldots
\end{array}\right|
$$

At last, one can write down the expression $\sum P(-t) P(t)$ again through Miwa variables:

$$
\sum_{k=0}^{n} P_{k+m}(-t) P_{n-k}(t)=\sum_{k=1}^{N} \frac{(-)^{n} \delta_{m, k}}{\Delta(\mu)} \operatorname{det}\left|\begin{array}{c}
\left\{\mu_{i}^{-n-1}\right\} \\
\left\{\mu_{i}^{0}\right\} \\
\ldots \\
\left\{\mu_{i}^{k-2}\right\} \\
\left\{\mu_{i}^{k}\right\} \\
\ldots
\end{array}\right|
$$

The last expression can be obtained from (13) by substituition $\lambda_{i} \rightarrow \mu_{i}^{-1}$. Then, the dependence of $N$ can be got rid of and the result is the determinant with the lines $\mu_{j}^{-m_{i}+i-1}$ such that $j$ goes from 1 to infinity and $m_{i} \neq 0$ only for $i \ll \infty$. Thus, we produce all the expressions for characters like above. The last thing is still remained is to prove the last formula. We can do this in the standard framework of [3] regularizing the expression for basis vector to have non-zero vacuum expectation value of the element of $G L(\infty)$. Namely, let us consider the renormalized Clifford element $\mathcal{G}$ (let $m \geq 1 ; n \geq 0)$ :

$$
\mathcal{G}^{(m, n)}=1+\left(A_{1} \psi_{-m}^{*}+A_{2} \psi_{n}^{*}\right)\left(B_{1} \psi_{-m}+B_{2} \psi_{n}\right)
$$

such that

$$
\mathcal{G}^{(m, n)}|0\rangle=\left(1+A_{2} B_{2}+A_{1} B_{2} \psi_{-m}^{*} \psi_{n}\right)|0\rangle
$$

and, for definiteness, let us choose $A_{1} B_{2}=-1,1+A_{2} B_{2}=\epsilon$. Then basic vectors are defined in the standard manner (37)

$$
\phi_{i}^{(m, n)}=\left.\frac{1}{(i-1) !} \partial_{t}^{i-1} \frac{\left\langle 0\left|\exp \left[\sum \frac{J_{n}}{n}\left(\mu^{-n}-t^{n}\right)\right] \mathcal{G}^{(m, n)}\right| 0\right\rangle}{(1-\mu t)\left\langle 0\left|\mathcal{G}^{(m, n)}\right| 0\right\rangle}\right|_{t=0} .
$$


Since in our case $\langle 0|\mathcal{G}| 0\rangle=\epsilon$ and

$$
\frac{\left\langle 0\left|\exp \left[\sum \frac{J_{n}}{n}\left(\mu^{-n}-t^{n}\right)\right] \mathcal{G}^{(m, n)}\right| 0\right\rangle}{\left\langle 0\left|\mathcal{G}^{(m, n)}\right| 0\right\rangle}=1+\frac{1}{\epsilon} \mu^{-n-1} t^{m-1}(1-\mu t),
$$

it is obvious that

$$
\phi_{i}^{(m, n)}=\mu^{i-1}+\frac{1}{\epsilon} \mu^{-n-1} \delta_{m i} .
$$

Therefore, the basic vectors are singular in the limit $\epsilon \rightarrow 0$, but from normalized $\tau$ - function we can obtain that the limit of the "Japanese" correlator

$$
\left\langle 0\left|\exp \left[\sum J_{n} T_{n}\right] \mathcal{G}^{(m, n)}\right| 0\right\rangle=\epsilon \frac{\operatorname{det}\left[\psi_{i}^{(m, n)}\left(\mu_{j}\right)\right]}{\Delta(\mu)}
$$

has well-defined limit when $\epsilon \rightarrow 0$. For example, in the case of two Miwa variables $(N=2)$ (it is trivial to check it for general case and reproduce proper expression) from eq.(6)

$$
\begin{array}{r}
\left\langle 0\left|\exp \left[\sum \frac{J_{n}}{n}\left(\mu_{1}^{-n}-\mu_{2}^{-n}\right)\right] \mathcal{G}^{(m, n)}\right| 0\right\rangle= \\
=\epsilon+\frac{1}{\Delta(\mu)}\left\{\delta_{m, 1}\left|\begin{array}{cc}
\mu_{1}^{-n-1} & \mu_{2}^{-n-1} \\
\mu_{1} & \mu_{2}
\end{array}\right|-\delta_{m, 2}\left|\begin{array}{cc}
\mu_{1}^{-n-1} & \mu_{2}^{-n-1} \\
1 & 1
\end{array}\right|\right\}
\end{array}
$$

It coincides with expression (49) and stress that it is impossible to do the regularization procedure under the determinant (i.e. to regularize just the basis vectors giving the point of the Grassmannian).

Now let us stress that we determined the determinant form of the singular $\tau$-function. The crucial difference with the regular case is the possibility to "skip" some vectors $\phi_{i}(\mu) \sim$ $\mu^{i-1}+O(1 / \mu)$ in the determinant (see the next subsection). The total lenght of the skipped "gap" $S$ is an important parameter [32]. It determines, in particular, the asymptotics of singular $\tau$-functions at small $t_{1}$ when all other times are equal to zero:

$$
\tau\left(t_{1}\right) \sim t_{1}^{S}
$$

\subsection{Character expansion of generic $\tau$-function}

Now we would like to discuss more on the statement iii) to involve it into the framework of generic KP $\tau$-functions. To start, let us consider an arbitrary $\tau$-function in the determinant form (36) with some vectors 


$$
\phi_{i}=\mu^{i} \sum_{k \geq 1} p_{i k} \mu^{-k}
$$

Let us consider for a while the determinant (36) of the finite size $N \times N$. Then, after permuting the columns in the determinant and multiplying this by $\mu^{-N}$, one can get

$$
\tau=\mu^{N} \frac{\operatorname{det} \bar{\phi}_{i}\left(\mu_{j}\right)}{\Delta(\mu)}
$$

where

$$
\bar{\phi}_{i}(\mu) \equiv \sum_{k=1} \bar{p}_{i k} \mu^{-k}
$$

and

$$
\bar{p}_{i j} \equiv p_{N-i+1, j-i+1}, \quad p_{i j} \equiv 0 \text { for } j<1 .
$$

Then we use the formula

$$
\operatorname{det}_{i j}\left(\sum_{k=1}^{\infty} f_{i k} p_{j k}\right)=\sum_{1 \leq k_{1}<k_{2}<\ldots}^{\infty} \operatorname{det}_{i j} f_{i k_{j}} \operatorname{det}_{i j} p_{i k_{j}}
$$

to rewrite $\tau$-function (59) as a sum

$$
\begin{gathered}
\tau=\sum_{1<k_{1}<k_{2}<\ldots}^{\infty} \operatorname{det}_{i j} \bar{p}_{i, k_{j}} \frac{\operatorname{det}_{i j} \mu_{i}^{N-k_{j}-1}}{\Delta(\mu)}= \\
=\sum_{m_{1} \geq m_{2} \geq \ldots \geq 0}^{\infty} \operatorname{det}_{i j} p_{i, m_{j}+i-j+1} \frac{\operatorname{det}_{i j} \mu_{i}^{-m_{j}+j-1}}{\Delta(\mu)},
\end{gathered}
$$

and we replaced $k_{i} \longrightarrow m_{N-i+1}+N-i+1$ (compare with (10) and (14)-(15)) and reflected the second index in the last determinant $i \longrightarrow N-i$ and analogously both indices in the first determinant.

One can observe that expression (7) is nothing but the linear combination of the characters (like (49)) discussed in the previous subsections. Certainly, it had to be evident from the very beginning that any $\tau$-function can be expanded into a sum of the characters as, in Miwa variables, any $\tau$-function is a symmetrical function (to be a function of times) and the characters of groups $G L(N)$ (= all possible Young tables) span the space of such functions. Thus, the only question to be addressed to is what are the coefficients $P\left\{m_{i}\right\}$ 's of the expansion 


$$
\tau=\sum_{m_{1} \geq m_{2} \geq \ldots \geq 0}^{\infty} P\left\{m_{i}\right\} \chi_{\left\{m_{i}\right\}}(G) .
$$

In fact, one can just demand for the $\tau$-function to satisfy the Hirota bilinear equations. Then, it can be demonstrated [33] that it means: the coefficients $P\left\{k_{i}\right\}$ 's should be the Plucker coordinates in the infinite dimensional Grassmannian. In its turn, this just implies that they can be presented in the determinant form

$$
P\left\{m_{i}\right\}=\operatorname{det}_{i j} p_{i, m_{j}+i-j+1}
$$

Thus, we are led to formula (36), there being no any restrictions to the asymptotics of the vectors $\phi_{i}(\mu)$.

Now, one can see immediately from (65) that, if some $m_{k+1}=0$ (and, therefore, so all $\left.m_{p}, p>k\right)$, then $P\left\{m_{i}\right\}$ will be determinant of block matrix:

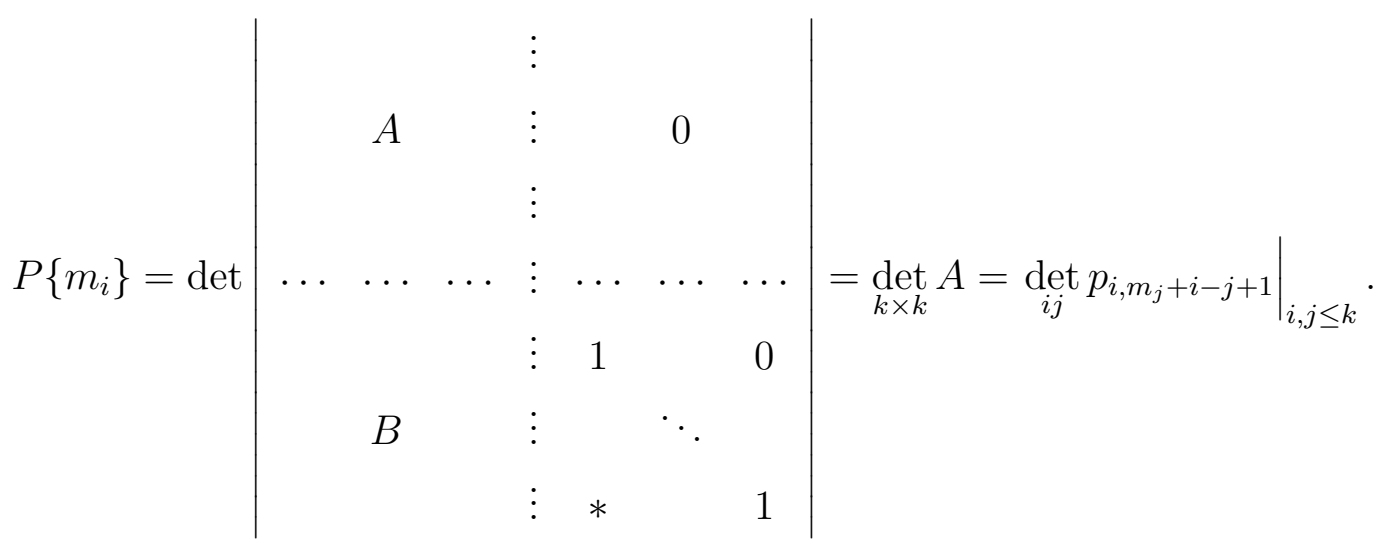

Thus, we obtain that the final answer for the expansion (64) does not depend on $N$ (certainly not be this would implies impossibility to rewrite the expansion (64) in terms of time variables), in spite of it was impossible to avoid introducing $N$ for the calculations. The only restriction to $N$ is that it should be larger than the number of non-zero $m_{i}$ 's, i.e. there should exist such $k \leq N$ that the representation with these $m_{i}$ 's can be embedded into the group $G L(k)$.

For the future use, let us remark that given some $P\left\{m_{i}\right\}$ one can always construct a whole family of solutions:

$$
P_{V}\left\{m_{i}\right\}=e^{\sum_{i} V\left(m_{i}\right)} P\left\{m_{i}\right\}
$$


with any function $V(x)$ (this corresponds to the change of $p_{i k}$ for $\left.e^{V(k)} p_{i k}\right)$.

To conclude the section, let us reformulate more precisely the statement iii). In fact, from (58)- 65) one can see that the $\tau$-function is singular, i.e. there are gaps in its determinant representation (36) when at least one of the coefficients $p_{i 1}$ in (58) is equal to zero. Actually, singular $\tau$-function is implied to be zero at all times equal to zero, i.e. when all $\mu_{i}$ in (63) are infinite. At these values of $\mu_{i}$ 's, the only trivial character (when all $m_{i}=0$ in (63)) does contributes to the sum (64). Therefore, $\tau$-function is singular, whenever the trivial character does not contribute, i.e. (see (64) and (65)) $P\left\{m_{i}=0\right\}=\operatorname{det}_{i j} p_{i, i-j+1}=\prod_{i} p_{i 1}=0$ (note that the matrix $p_{i, i-j+1}$ is upper-triangle - see ([1)).

Thus, we proved that

iii') Arbitray $\tau$-function can be expanded to an (infinite) sum of the characters of groups $G L(N)$ (13) or (16), the $\tau$-function being singular if and only if at least one of $p_{i 1}=0$.

This completes our consideration of the connection between (singular) $\tau$-functions and characters of groups $G L(N)$.

\section{Bilinear combination of characters}

An obvious particular example of Plucker function $P_{R}=P\left\{k_{i}\right\}$ of the form (65) is provided by characters themselves, $P_{R} \sim \chi_{R}\left(\bar{G}^{-1}\right)$ with some element $\bar{G}$. Making use of the freedom (67) we conclude that

$$
\begin{gathered}
K(G, \bar{G}) \equiv \sum_{1 \leq k_{1}<k_{2}<\ldots}^{\infty} \chi_{\left\{k_{i}\right\}}(G) \chi_{\left\{k_{i}\right\}}\left(\bar{G}^{-1}\right) e^{\sum_{i} V\left(k_{i}\right)}= \\
=\sum_{R} \chi_{R}(G) \chi_{R}\left(\bar{G}^{-1}\right) e^{V(R)}
\end{gathered}
$$

is always a KP $\tau$-function as a function of time variables $t_{k}=\frac{1}{k} \operatorname{tr} G^{k}=\frac{1}{k} \sum_{i} \lambda_{i}^{k}=\frac{1}{k} \sum_{i} \mu_{i}^{-k}$ 田

In order to construct $\tau$-function of Toda lattice hierarchy one should introduce somehow the additional set of the independent parameters (negative times). In formula (68) these parameters are $\bar{t}_{m}=\frac{1}{m} \operatorname{tr} \bar{G}^{-k}=\frac{1}{k} \sum_{i} \bar{\lambda}_{i}^{-k}=\frac{1}{k} \sum_{i} \xi_{i}^{k}$. We establish below that a particular bilinear combinations of characters (68) does really solve to Toda lattice hierarchy.

\footnotetext{
${ }^{11}$ In fact, we have not chosen the normalization of this $\tau$-function yet. We fix it to be $\tau(0)=1$, which sometimes requires some trivial renormalization of $K(G, \bar{G})$.
} 
The simplest example of such solution which has the apparent group-theoretical meaning is $e^{V(k)}=\frac{1}{k !}$. Then

$$
\tau(t, \bar{t})=\frac{\operatorname{det} \exp \left[\lambda_{i} \xi_{j}\right]}{\Delta(\lambda) \Delta(\xi)}
$$

is nothing but Itzykson-Zuber formula (四).

We can immediately calculate the basic vectors using formulas of section 5.4. After proper normalization these are

$$
\phi_{i}(\mu)=\mu^{i-1} \sum_{k=0}^{\infty} e^{V(k+N-1)-V(N-i)} P_{k}(\bar{t}) \mu^{-k \mu \gg 1} \mu^{i-1}\left(1-O\left(\mu^{-1}\right) .\right.
$$

Let us discuss properties of expression (68) as the $\tau$-function. In particular, we will find an explicit representation of the corresponding Clifford element in the fermionic correlator (26). One can consider this element as parametrized by the whole set of the negative times; in this case correlator takes the form

$$
\tau_{n}(t, \bar{t}) \equiv\left\langle n\left|e^{H(t)} \mathcal{G}(\bar{t}),\right| n\right\rangle
$$

where

$$
\mathcal{G}(\bar{t}) \equiv \mathcal{G} \times e^{\bar{H}(\bar{t})}
$$

Therefore, in Miwa variables, one can obtain the determinant representation (36) where the basic vectors are now the functions of $\{\bar{t}\}$ :

$$
\tau_{n}(t, \bar{t})=\langle n|\mathcal{G}(\bar{t})| n\rangle \frac{\operatorname{det} \phi_{i}\left(\mu_{j}\right)}{\Delta(\mu)}
$$

where $\phi_{i}(\mu)$ have the following form:

$$
\phi_{i}^{(c a n)}(\mu)=\mu^{n-1} \frac{\left\langle n\left|\psi_{n-i} \psi^{*}(\mu) \mathcal{G}(\bar{t})\right| n\right\rangle}{\langle n|\mathcal{G}(\bar{t})| n\rangle} .
$$

Here superscript "can" means that these vectors has the canonical form:

$$
\phi_{i}^{(\text {can })}(\mu)=\mu^{i-1}+\sum_{k=0}^{\infty} \mu^{k-1} \frac{\left\langle n\left|\psi_{n-i} \psi_{n+k}^{*} \mathcal{G}(\bar{t})\right| n\right\rangle}{\langle n|\mathcal{G}(\bar{t})| n\rangle} .
$$

In order to reproduce (70) one can use a very simple (non-ordered !) Clifford element which describes completely the point of Grassmannian which corresponds to "group-theoretical" $\tau$ function (68):

$$
\mathcal{G}=\exp \left\{\sum_{k \in Z} V_{k} \psi_{k} \psi_{k}^{*}\right\} \equiv \prod_{k \in Z} \mathcal{G}^{(k)}
$$


where

$$
\mathcal{G}^{(k)} \equiv e^{V_{k} \psi_{k} \psi_{k}^{*}}=1+\left(e^{V_{k}}-1\right) \psi_{k} \psi_{k}^{*} .
$$

Then it is easy to see that fermionic modes are transformed under the action of this Clifford element almost trivially

$$
\mathcal{G} \psi_{k} \mathcal{G}^{-1}=e^{V_{k}} \psi_{k}, \mathcal{G} \psi_{k}^{*} \mathcal{G}^{-1}=e^{-V_{k}} \psi_{k}^{*}
$$

and in this case the canonical basic vectors (75) have the form:

$$
\phi_{i}^{(c a n)}(\mu)=\sum_{k=0}^{\infty} \mu^{i+k-1} e^{\left[V_{n-i+k}-V_{n-i}\right]} \sum_{m=0}^{i-1} P_{m}(-\bar{t}) P_{k-m}(\bar{t}) .
$$

One can show that combining columns in the determinant it is possible to obtain the new (non-canonical) basis

$$
\phi_{i}(\mu)=\sum_{k=0}^{\infty} \mu^{i+k-1} e^{\left[V_{k+n-i}-V_{n-i}\right]} P_{k}(\bar{t}),
$$

since these vectors are linear combinations of the canonical ones:

$$
\phi_{i}(\mu)=\sum_{k=1}^{i} P_{i-k}(\bar{t}) e^{\left[V_{n-k}-V_{n-i}\right]} \phi_{k}^{(c a n)}(\mu) .
$$

The vectors (80) coincide with (80), the parameter $N$ playing the role of zero time $n$. It means that we really have demonstrated that formula (68) is the $\tau$-function of Toda lattice hierarchy.

As the simplest example of the above construction, for the trivial element $\mathcal{G}=1 \tau$-function (71) has well-known form

$$
\tau_{n}(t, \bar{t})=\exp \left\{\sum_{k=1}^{\infty} k t_{k} \bar{t}_{k}\right\}
$$

and (non-canonical) basic vectors are:

$$
\phi_{i}(\mu)=\mu^{i-1} \exp \left\{\sum_{k=1}^{\infty} \bar{t}_{k} \mu^{-k}\right\} .
$$

Strictly speaking, the element (76) has (for some particular choises of $V_{n}$ ) the infinite (or, vice versa, zero) norm since

$$
\mathcal{G}|n\rangle=\prod_{k=-\infty}^{-1} e^{V_{k}}|n\rangle .
$$

This is of great importance, however, since the ratio $\tau(t, \bar{t}) /\langle n|\mathcal{G}(\bar{t})| n\rangle$ in (73) as well as the ratio in (75) is finite. To avoid all the troubles, one can trivially observe that there 
exists normally ordered element (we use the first normal ordering of section 5) with the same transformation properties as in (78) which has finite norm from the very beginning:

$$
\tilde{\mathcal{G}}=\ddagger \exp \left\{\sum_{k<0}\left(1-e^{-V_{k}}\right) \psi_{k} \psi_{k}^{*}+\sum_{k \geq 0}\left(e^{V_{k}}-1\right) \psi_{k} \psi_{k}^{*}\right\} \ddagger
$$

and

$$
\begin{gathered}
\tilde{\mathcal{G}}|n\rangle=\frac{\prod_{k=-\infty}^{n} e^{V_{k}}}{\prod_{k=-\infty}^{-1} e^{V_{k}}}|n\rangle \equiv \\
\equiv \begin{cases}\prod_{k=0}^{n} e^{V_{k}}|n\rangle & n \geq 0 \\
\prod_{k=n}^{-1} e^{-V_{k}}|n\rangle & n<0\end{cases}
\end{gathered}
$$

Two elements (76) and (85) are related by the obvious expression:

$$
\mathcal{G}=\tilde{\mathcal{G}} \prod_{k=-\infty}^{0} e^{V_{k}}
$$

It is important to stress that (68) describes KP hierarchy w.r.t. time variables $t_{k}$ at any choices of the variables $\bar{t}_{k}$. In particular, the case of one hole correlator in $2 d$ YM theory (see section 3) corresponds to all $t_{k}=1$. In this case, the basis vectors (80) depend on the number of Miwa variables $N$. It would mean that such correlator is not any KP $\tau$-function, despite of the construction above. The puzzle is resolved by noting that the basis vectors depend only on the number of variables $\xi_{i}$, which describe negative times, and their number is equal to the number of $\lambda_{i}$ only by theoretical-group reasons.

Let us note now that the function $V(R)=\sum_{i} V\left(k_{i}\right)$ being represented in the form

$$
V(R)=\sum_{n=1}^{\infty} s_{n}\left(\sum_{i} k_{i}^{n}\right)
$$

is very similar to

$$
\tilde{V}(R)=\sum_{n=1}^{\infty} \tilde{s}_{n} C_{n}(R)
$$

where $C_{n}(R)$ is the value of $n$-th Casimir operator in representation $R$. However, the exact form of $\tilde{V}(R)$ depends on the choice of Casimir operators. Canonical choice, related to the equation

$$
\Delta_{n} \chi_{R}=C_{n}^{c}(R) \chi_{R}
$$

is:

$$
C_{n}^{c}(R)=\operatorname{tr}_{R} E_{i_{1} i_{2}} \ldots E_{i_{n} i_{1}}
$$


and does not provide $C_{n}(R)$ in the form, necessary for (68). Operators defined so that

$$
C_{n}^{f}(R)=\sum_{i} k_{i}^{n}
$$

are also Casimir operators (they are non-linear functions of $C_{n}^{c}(R)$ ), they are suitable for the use in (68), but they do not arise in eq.(90). The special case is quadratic Casimir operator for $S L$ group $(\operatorname{not} G L): C_{2}^{f}(R)-C_{2}^{c}(R)=$ const.

Formula (68) arises as partition function of $G(K M)^{2}$ with one site $\alpha$ and two external fields $L$ and $\bar{L}, G=e^{L}, \bar{G}=e^{\bar{L}}$, if it is considered as "unitary" model (i.e with sums over integer eigenvalues of $\Phi$ instead of integrals). It also arises as correlator of two Wilson loops on a sphere in the $2 d$ Yang-Mills theory [12, 26], in this case usually $V(R)=s C_{2}^{c}(R)$ (for generic potential $V_{R}$ one can speak about "generalized $2 d$ Yang-Mills model"). We see that it is better to define it with potential $V(R)$ rather than $\tilde{V}(R)$ : then this quantity is KP $\tau$-function. Moreover, it is automatically a Toda-lattice $\tau$-function, if "negative times" are introduced as $\bar{t}_{k}=\frac{1}{k} \operatorname{tr} \bar{G}^{-k}$.

\section{Comments on Kontsevich model}

Eq.(68) naturally arises from "unitary" (i.e. discretized) Kontsevich model with single site $\alpha$ and two background fields. A slightly more general model with $m$ background fields is

$$
\begin{aligned}
\mathcal{F}_{V}\{L\} & \equiv \frac{\mathcal{Z}_{V}\{L\}}{\mathcal{C}_{V}\{L\}}=\int d \Phi \prod_{\mu=1}^{m}\left[d U_{\mu}\right] e^{\operatorname{tr} V(\Phi)} \prod_{\mu=1}^{m} e^{\operatorname{tr} L_{\mu} U_{\mu} \Phi U_{\mu}^{\dagger}}= \\
& =\prod_{i} \int d \phi_{i} e^{V\left(\phi_{i}\right)} \Delta(\phi)^{2-m} \prod_{\mu=1}^{m} \hat{\chi}_{\left\{\phi_{i}\right\}}\left(e^{L_{\mu}}\right),
\end{aligned}
$$

where $\hat{\chi}_{\left\{\phi_{i}\right\}}\left(e^{L_{\mu}}\right)=\frac{\Delta\left(e^{L}\right)}{\Delta(L)} \chi_{\left\{\phi_{i}\right\}}\left(e^{L_{\mu}}\right)$. The sewing operation, i.e. integration over $g$ pairs of background fields with the weight $\delta\left(L_{1}+L_{2}\right) d L_{1} d L_{2}$ gives:

$$
\mathcal{F}_{V}\{L\}=\prod_{i} \int d \phi_{i} e^{V\left(\phi_{i}\right)} \Delta(\phi)^{2-2 g-m} \prod_{\mu=1}^{m} \hat{\chi}_{\left\{\phi_{i}\right\}}\left(e^{L_{\mu}}\right),
$$

The discretized ("unitary") version of the model is 


$$
\begin{aligned}
\mathcal{F}_{V}^{d s c r}\{L\}= & \prod_{i} \sum_{\phi_{i} \in Z_{+}} e^{V\left(\phi_{i}\right)} \Delta(\phi)^{2-2 g-m} \prod_{\mu=1}^{m} \hat{\chi}_{\left\{\phi_{i}\right\}}\left(e^{L_{\mu}}\right)= \\
& =\sum_{R} e^{V(R)} d_{R}^{2-2 g-m} \prod_{i} \hat{\chi}_{R}\left(e^{L_{i}}\right),
\end{aligned}
$$

An important feature of the discrete sum is that it is defined over model space and thus eigenvalues $\phi_{i}$ are not only restricted to be integers, but also they should be non-negative integers. This makes the discretized model essentially different from continuous Kontsevich model. Namely, in the case of continuous GKM one could usually rely upon quasiclassical (steepest descent) calculation, which gives rise to expansion of the integral in powers of $m_{i}^{-1}$ with matrix $M$ related to $L$ as $V^{\prime}(M)=L$. However, this regime can be forbidden in discretized model, if the stable point of the action lies in the domain of negative $\phi_{i}$ (it depends on the potential). This implies that besides the Kontsevich-type "phase" the discretized model has also another - simpler - phase, where the natural expansion is just in powers of $G_{\mu}=e^{L_{\mu}}$. We refer to it as to "character phase". It is in this phase that our reasoning from the previous section was actually applicable and this was why the point of Grassmannian appeared so simple.

The study of Kontsevich phase is more complicated. Indeed, it corresponds to modified reduction condition (having now non-local form) and the Ward identity which usually fixes the string equation contains now some boundary terms. But we would like to stress that in this phase the basis vectors for the KP case (in contrast to the character phase) do not depend on $N$ from the very beginning (the reason for this is that the point of the Grassmannian given by $\mathcal{G}(\bar{t})(\overline{72})$ does not depend on the number of Miwa variables $\xi_{i}$ when all times $t_{k}=1$ ).

In character phase the quantities (95) are $\tau$-functions only for $g=0$ and $m=1,2$. What happens in other cases can be illustrated by the following simple example. Take $m=2$ and $V=0$, i.e. $\mathcal{G}=1$ (see (82)). Let us begin from the $1 \times 1$ matrices. Then we always have (for any $g$ )

$$
d \lambda^{\frac{1}{2}} d \bar{\lambda}^{-\frac{1}{2}} K=d \lambda^{\frac{1}{2}} d \bar{\lambda}^{-\frac{1}{2}} \sum_{k=0}^{\infty} \lambda^{k} \bar{\lambda}^{-k}=\frac{d \lambda^{\frac{1}{2}} d \bar{\lambda}^{\frac{1}{2}}}{\lambda-\bar{\lambda}}=\left\langle 0\left|\psi(\lambda) \psi^{*}(\bar{\lambda})\right| 0\right\rangle=\Psi(\lambda, \bar{\lambda}) .
$$

Now we have a simple prescription (40) to define the quantity for $N \times N$ matrices so that it automatically becomes $\tau$-function (certainly, this is equivalent to formula (82) when is 
rewritten in Miwa variable):

$$
K_{N}=\frac{\operatorname{det}_{i j} K_{1}\left(\lambda_{i}, \bar{\lambda}_{j}\right)}{\Delta(\lambda) \Delta(\bar{\lambda})}=\frac{\prod_{i} \bar{\lambda}_{i}}{\prod_{i<j}\left(\lambda_{i}-\bar{\lambda}_{j}\right)}=\frac{\Psi\left(\lambda_{i}, \bar{\lambda}_{j}\right)}{\Delta(\lambda) \Delta(\bar{\lambda})},
$$

where we used the formula for the Cauchy determinant:

$$
\operatorname{det}_{i j} \frac{1}{x_{i}-y_{j}}=\frac{\Delta(x) \Delta(y)}{\prod_{i, j}\left(x_{i}-y_{j}\right)} .
$$

One can easily see that this leads to the formula with $g=0$ (and non-vanishing potential can be easily introduced, as explained in the previous section).

This is already enough to conclude that expression for $g>0$ are not $\tau$-functions, simply because they are different from the one arising by the above rule. To see what they look like let us consider an example of $N=2$ (still with $V=0$ and $m=2$ ). Then we have:

$$
\begin{gathered}
K_{2}=\sum_{k_{2}>k_{1} \geq 0}^{\infty} \frac{\left(\lambda_{1}^{k_{1}} \lambda_{2}^{k_{2}}-\lambda_{1}^{k_{2}} \lambda_{2}^{k_{1}}\right)\left(\bar{\lambda}_{1}^{k_{1}} \bar{\lambda}_{2}^{k_{2}}-\bar{\lambda}_{1}^{k_{2}} \bar{\lambda}_{2}^{k_{1}}\right)}{\left(k_{1}-k_{2}\right)^{2 g}}= \\
=\frac{\bar{\lambda}_{1} \bar{\lambda}_{2}}{\lambda_{1} \lambda_{2}-\bar{\lambda}_{1} \bar{\lambda}_{2}}\left[L i_{2 g}\left(\frac{\lambda_{1}}{\bar{\lambda}_{1}}\right)+L i_{2 g}\left(\frac{\lambda_{2}}{\bar{\lambda}_{2}}\right)-L i_{2 g}\left(\frac{\lambda_{1}}{\bar{\lambda}_{2}}\right)-L i_{2 g}\left(\frac{\lambda_{2}}{\bar{\lambda}_{1}}\right)\right],
\end{gathered}
$$

where $L i_{p}[x] \equiv \sum_{i=1}^{\infty} \frac{x^{i}}{i^{p}}$ is nothing but polylogarithm function. It can be also re-written as

$$
K_{2}=\int^{1} \frac{d z_{2 g}}{z_{2 g}} \ldots \int^{z_{3}} \frac{d z_{1}}{z_{2}} \int^{z_{2}} \frac{d z_{1}}{z_{1}} \operatorname{det}\left|\begin{array}{cc}
\Psi\left(\lambda_{1}, z_{1} \bar{\lambda}_{1}\right) & \Psi\left(\lambda_{1}, \frac{1}{z_{1}} \bar{\lambda}_{2}\right) \\
\Psi\left(\lambda_{2}, z_{1} \bar{\lambda}_{1}\right) & \Psi\left(\lambda_{2}, \frac{1}{z_{1}} \bar{\lambda}_{2}\right)
\end{array}\right|
$$

i.e. for the case of generic $N$

$$
K_{N}=\left.J \frac{\operatorname{det} \Psi\left(\lambda_{i}, z_{j} \bar{\lambda}_{j}\right)}{\Delta(\lambda) \Delta(\bar{\lambda})}\right|_{z_{i j}=1}
$$

Here $z_{j}=\prod_{k \neq j} z_{j k}, z_{k j}=z_{j k}^{-1}$ and $J$ is an integral operation $J=\prod_{i<j} J_{z_{i j}}^{2 g}, J_{z}=\int \frac{d z}{z}$, where integral is defined so that $J_{z} z^{p}=\frac{z^{p}}{p}$ for any $p$.

In other words, for $g \neq 0$ in the character phase we get integrals of $\tau$-functions. Indeed, it is not so suprising and one can trivially understand this already for the case of multi-point correlators in the sphere (see (95)). It is sufficient to use the identity for characters:

$$
\chi_{\alpha}\left(G_{1}\right) \chi_{\alpha}\left(G_{2}\right)=d_{\alpha} \int[d U] \chi_{\alpha}\left[G_{1} U G_{2} U\right]
$$


to reduce the arbitrary $n$-point correlator to $(n-2)$-integrated $\tau$-function of 2TDL hierarchy. In this case, similar to eq.(101), the integrations go over the variables which "rotate" the group element $G$ giving Miwa times. We do not know what does it mean in the integrability framework.

\section{Concluding remarks}

The simple manipulations, described in the previous subsection, raise many puzzling problems, which can stimulate new investigation and after all provide a new view on the entire theory of matrix models and integrability. Now we want to mention some of these problems somewhat more explicitly.

Nature of variables in the $G(K M)^{2}$

Once external (background) fields are introduced as in (1), one is naturally led to interpretation of integration over eigenvalues of $\Phi^{(\alpha)}$-fields as that over weight space of $G L$ group. This weight space can be also called the space of all represntations, i.e. a model space. This gives rise to a natural suggestion to consider a "unitary-group" version of the model, where integration is substituted by a sum over irreducible representations of unitary groups, i.e. over Young diagramms. Then $G(K M)^{2}$ includes integration over model-valued fields, and what is lacking is the group-theoretical meaning (if any) of the model (11), which implies a somewhat specific interaction between different model-variables $\Phi^{(\alpha)}$. This problem is of course absent in the case of a single point $\alpha$, i.e. for Kontsevich model with several external fields, which can be in fact sufficient for the study of the generalized $2 d$ Yang-Mills and sum other theories.

Alternatively one could think that $e^{\Phi^{(\alpha)}}$ should be interpreted as elements of unitary group rather then label representations. This is a different view, since it implies that eigenvalues of $\Phi^{(\alpha)}$ lie on a segment $[0,2 \pi]$ which is treated as compactified circle. One of the problems with this interpretation is that it seems to require some periodicity under the shift of any eigenvalue of $\Phi^{(\alpha)}$ by $2 \pi$, which is apparently absent in Lagrangain of the model (何). Eq.(雨) could serve as an illustration that this periodicity (for eigenvalues of $L$ ) can be restored after integration over $U$ (provided eigenvalues of $X$ are integers, i.e. for representations of unitary group), however eq.(2) seems to imply that this does not need to be the case in the $G(K M)^{2}$, as defined in 
eq.(1). An even more severe problem is apparent non-priodicity of potential term $\operatorname{tr} V(\Phi)$ for polinomial $V$."2 Because of these problems we prefer the above interpretation of $\Phi^{(\alpha)}$ 's as elements of the weight (model) space, when $V(\Phi)$ has a natural interpretation in terms of Casimir operators. The alternative picture with unitary $e^{\Phi}$ can arise after certain "Fourier transform" of the model (1), which remains to be worked out (it has very good chances to be similar to the same model (1) in the Gaussian case of quadratic potential $V(\Phi) \sim \Phi^{2}$.)

\section{Integrability properties}

Conceptually integrability is usually related to correlation functions of (any kind of) free fields (i.e. allowing for Wick theorem) on (any kind of) Riemann surfaces: such correlators can be always interpreted as $\tau$-functions in Miwa parametrization (usually one considers only free-fermion $\tau$-function, related to level $k=1$ Kac-Moody algebras, but this should not be a serious restriction ${ }^{\mathbb{1 3}}$ ). However, amplitudes in topological and string models are usually integrals of such correlation functions over some moduli (of Riemann surfaces or bundles over Riemann surfaces), essentially over whole Universal Moduli Space, but not over its subspaces corresponding to finite genus Riemann surfaces. Thus, generic quantities of interest in this framework are integrals of $\tau$-functions (unless in some special circumstances like (68) and (97) where integrals accidently are not present). Adequate group-theory (or orbit-theory) interpretation of integrals of $\tau$-functions (similar to that in [34] for $\tau$-functions themselves) remains to be found.

This is a trivial remark, what deserves emphasising is that from certain point of view it is a step back from the main achievement of matrix model theory. Namely, matrix models were used to prove that exact partition functions, if considered as functions of coupling constants (i.e. as functions on the spaace of string models or of moduli of bundles over Riemann surfaces) are $\tau$-functions, i.e. these were $\tau$-functions arising as correlators in spectral space, not on the world surfaces, and thus the above argument did not necessarily implied ocuurence of integrals of $\tau$-functions. Remarkably, in the framework of Kontsevich models dependence on the string model could be imitated in effective way by that on the matrix external field $L$. Somehow, we see that this is no longer true for the $G(K M)^{2}$ : one should abandon at least one

\footnotetext{
12 There is some discussion of this issue in a recent paper [18] in the specific case of quadratic potential.

${ }^{13}$ Such restriction is an explicit consequence of requirement that integrable flows commute instead of forming some closed non-abelian algebra.
} 
of the two suggestions: (a) that $G(K M)^{2}$ is a natural generalization of GKM for description of more sophisticated string models and/or (b) that non-perturbative partition functions are usually $\tau$-functions. Option (a) is for sure true, at least, in the sense that $G(K M)^{2}$ is still not general enough. Most probably both suggestions deserve serious modifications, but the study of $G(K M)^{2}$ can shed more light on this problem.

\section{Specifics of Kontsevich model}

There is certain difference between the ways in which Miwa variables arise in GKM and in our considerations of the Kontsevich phase in this paper. In group (character) theory the time-variables are usually represented as $t_{k}=\frac{1}{k} \operatorname{tr}_{F} G^{k}=\frac{1}{k} \operatorname{tr} e^{k L}$, while in GKM $t_{k}=\frac{1}{k} \operatorname{tr} M^{-k}$, where $M$ is related to $L$ in potential-dependent way: $V^{\prime}(M)=L$. This difference is because in the context of GKM the time-variables appear in quasiclassical expansion of partition function around a stable point $\Phi=L$ in the integral (2), while in character theory the idea is that every particular character is by itself a polinomial in $t$-variables. These two approaches are in a sense orthogonal. The correspondence can be restored by a change of reasoning in character theory, as described in the section 2 above.

Another possibility is to consider specific potential $V_{0}(\Phi)=-\Phi \log \Phi$, when $L=V_{0}^{\prime}(M)=$ $-\log M$, and the two descriptions literally coincide. This is a remarkable version of GKM for many reasons, of which we mention only two. The simplest heuristic remark is that it should describe the $(p, 1)$ model with $p=+0$, i.e. with $c=1-\frac{6(p-q)^{2}}{p q}=-\infty$, which has good chances to be related to some simple physical theories, including $2 d$ Yang-Mills. This argument seems not least convincing than the one distinguishing GKM with potential $V_{-1}(\Phi)=\log \Phi(p=-1)$ as the one describing (a part of) the $c=25$ (i.e. essentially $c=1$ ) string model, which is partly confirmed by explicit investigation in [35, 36, 8]. Both potentials $V_{0}$ and $V_{-1}$ are beyond the region of $p>1$ where quasiclassical treatement of GKM is valid, and there are certain corrections to standard GKM theory. The second remark is that these potentials are very similar to two remarkable choices: $V_{\Gamma}(\Phi)=-\log \Gamma(\Phi)$ and $V_{\psi}(\Phi)=\log \psi(\Phi)$ respectively (here $\Gamma(x)$ is Euler Gamma-function and $\psi(x)=\frac{\partial}{\partial x} \log \Gamma(x)$. These are distinguished because $e^{V_{\Gamma}(x)}=\frac{1}{\Gamma(x)}$ and $e^{V_{\psi}(x)}=\psi(x)=\sum_{k=0}^{\infty} \frac{1}{x+k}$, and for special choices of integration contours (around the singularities at negative $x$ ) the integrals over $\phi$ 's reduce to sums over discrete negative integer values of $\phi$ - this is exactly what necessary for going from non-compact 
version of (1) to the compact one. It deserves emphasizing that arising sums are not over all integers, but only over integers of one sign, as required for enumeration of Young tables (with non-negative rows lenghts) and irreducible representation of unitary groups.

\section{Generalization of characters}

Quantum mechanics on group manifolds and coadjoint orbits is not exhausted by formulas (4) and (9). There exist various matrix-like integral representations of matrix elements, more general than characters, namely, - for zonal spherical functions $\Phi_{\left\{k_{i}\right\}}(q)$. In particular, there are intriguing Harish-Chandra formulas like [37, 38] (we write down these for the zonal spherical functions on the symmetric spaces with the root system $A_{n-1}$ )

$$
\Phi_{\left\{k_{i}\right\}}(q)=\int_{u} d u \prod_{l=1}^{n-1} \Delta_{l}^{i \frac{K_{n-l+1}-K_{n-l}}{2 a}-m a}\left(u e^{2 a q} u^{-1}\right)
$$

where $q=\operatorname{diag}\left(q_{1}, \ldots, q_{n}\right), K=\left(K_{1}, \ldots, K_{n}\right), \Delta_{l}$ are the lowest corner minors of the matrix $u e^{2 a q} u^{-1}, u$ is a stationary subgroup of the corresponding symmetric space, $d u$ is invariant measure (normalized to 1 ) and $m$ is a number depending on the type of the symmetric space.

Moreover, it turns out, in certain cases zonal spherical functions possess determinantal representations, which allow to interpret them as $\tau$-functions, despite they are defined as matrix elements, not as partition functions. The simplest examples ${ }^{\mathbb{4}}$ are zonal spherical functions on the symmetric spaces of type $A I I I=S U(r, t) / S(U(r) \oplus U(t))$. They are labeled by a set of integers $\left\{k_{i}\right\}$ and given in the explicit detrminant form [39] (for the sake of brevity we write down the result only for the compact spaces):

$$
\Phi_{\left\{k_{i}\right\}}(q) \sim \frac{\operatorname{det}_{i j} F_{k_{i}}\left(\lambda_{j}\right)}{\Delta(\lambda) \Delta\left(k^{2}\right)}
$$

where $\lambda \equiv \cos 2 q$ in notations of [38], $F_{k_{i}}(\lambda)=P_{\left\{m_{i}\right\}}^{\alpha, \beta}(\cos 2 q)$ are Jacobi polynomials and $\alpha=\mu_{1}+\mu_{2}+\frac{1}{2}, \beta=\mu_{2}-\frac{1}{2}, m_{i}=\frac{1}{2} k_{i}-\frac{1}{2}\left(\mu_{1}+2 \mu_{2}\right)$. The constants $\mu_{1}$ and $\mu_{2}$ can be taken arbitrary though the group values are $\mu_{1}=2(r-s) ; r, s \in Z, \mu_{2}=2$. We do not dwell upon this interesting subject here, because its implications for the general theory remains obscure for us. At least it can be considered as an interesting application of DH-like theory and confirm the suggestion, that everything, arising from DH-theorem should have interpretation

\footnotetext{
${ }^{14}$ See 38 for more sophisticated examples.
} 
in terms of $\tau$-functions. We refer to [40] and [15] for some more details about this application (in [15] also a natural interpretation in terms of Calogero model is discussed).

Note on the formula $\sum_{R} e^{\sum s_{k} C_{k}(R)} \chi_{R}(G) \chi_{R}\left(\bar{G}^{-1}\right)$.

Let us mention one more similarity of this formula (68) to the standard GKM model.

Partition function of GKM is a $\tau$-function subjected to a set of additional equations. These equations form a set of Virasoro (or $W$-) constraints and their explicit appearance depends on the shape of potential function. Moreover, the derivatives w.r.t. the coefficients of the potential are expressed in a single way through time derivatives [10].

Analogous procedure can be performed in the model considered in this paper. Namely, one can differentiate (68) w.r.t. parameters $s_{n}$ of potential function (88). This gives rise to the Casimir eigenvalues $C_{n}$ in the sum (68):

$$
\frac{\partial}{\partial s_{n}} \sum_{R} e^{\sum t_{k} C_{k}(R)} \chi_{R}(G) \chi_{R}\left(\bar{G}^{-1}\right)=\sum_{R} C_{n}(R) e^{\sum t_{k} C_{k}(R)} \chi_{R}(G) \chi_{R}\left(\bar{G}^{-1}\right) .
$$

Alternatively, one can use (90) to represent the r.h.s. as result of the action of certain differential operators on the characters. Explicit expressions for these operators can be easily found in terms of $\lambda_{i}$-derivatives. After Miwa transformation, these can be finally rewritten as differential operators in times $t_{k}$ (in the spirit of [41]).

Certainly, to generate some analogs of Virasoro (or $W$ ) constraints, one also needs Ward identities. They should connect Casimirs and powers of Miwa variables and are more complicated than the usual ones [3] due to appearance of boundary terms (as the sum in (68) runs only over positive integers).

Having a set of constraints of Virasoro type, one can naturally compare the parameters $s_{n}$ with "potential times" of [10] as the r.h.s. of the eq.(105) can be usually obtained by taking derivatives in times $t_{n}$. Along with the constraint algebra it fixes the dependence of (68) both on times $t_{n}$ and on times $s_{n}$.

\section{Acknowledgements}

We benefited from the discussions with E.Antonov, A.Gerasimov, A.Gorsky, A.Losev, N.Nekrasov, A.Niemi, M.Olshanetsky, I.Polyubin, A.Zabrodin. 
The main part of this report was written during our stay at Institute of Theoretical Physics, Uppsala University, and we highly appreciate the hospitality and support. The work of S.K., A.Mar. and A.Mir. is partially supported by grant 93-02-14365 of the Russian Foundation of Fundamental Research.

\section{Appendix A. Fermionic language for $\tau$-functions}

\section{A1. Free fermions}

In this Appendix some notations and definitons on the fermionic representation of $\tau$-functions are collected. The main references are [3, 28, 30].

We start with the anticommutation relations for the fermionic modes:

$$
\left\{\psi_{i}, \psi_{j}^{*}\right\}=\delta_{i j}, \quad\left\{\psi_{i}, \psi_{j}\right\}=\left\{\psi_{i}^{*}, \psi_{j}^{*}\right\}=0
$$

Totally empty vacuum $|+\infty\rangle$ is defined by relations

$$
\psi_{i}|+\infty\rangle=0, \quad i \in Z
$$

Then the " $n$-th" vacuum is defined as follows:

$$
|n\rangle=\psi_{n}^{*} \psi_{n+1}^{*} \ldots|+\infty\rangle
$$

and satisfies the following conditions

$$
\psi_{k}^{*}|n\rangle=0 \quad k \geq n \quad, \quad \psi_{k}|n\rangle=0 \quad k<n
$$

Free fermionic fields

$$
\psi(z) \equiv \sum_{i \in Z} \psi_{i} z^{i} \quad, \quad \psi^{*}(z) \equiv \sum_{i \in Z} \psi_{i}^{*} z^{-i}
$$

can be bosonized with the help of the free bosonic field $\phi(z)$ :

$$
\begin{gathered}
\phi(z)=x-i p \log z+i \sum_{k \in Z} \frac{J_{k}}{k} z^{-k} \\
{[x, p]=i \quad ; \quad\left[J_{m}, J_{n}\right]=m \delta_{m+n, 0}}
\end{gathered}
$$


as follows

$$
\begin{gathered}
\psi(z)=: e^{i \phi(z)}: \equiv \\
\equiv e^{i x} e^{p \log z} \exp \left[\sum_{k=1}^{\infty} \frac{\bar{J}_{k}}{k} z^{k}\right] \times \exp \left[-\sum_{k=1}^{\infty} \frac{J_{k}}{k} z^{-k}\right] \\
\psi^{*}(z)=z: e^{-i \phi(z)}: \equiv \\
\equiv z e^{-i x} e^{-p \log z} \exp \left[-\sum_{i=1}^{\infty} \frac{\bar{J}_{k}}{k} z^{k}\right] \times \exp \left[\sum_{i=1}^{\infty} \frac{J_{k}}{k} z^{-k}\right]
\end{gathered}
$$

where

$$
J_{-k} \equiv \bar{J}_{k} \quad, \quad k>0
$$

Using definition eq.(A7) one can easily show that

$$
: e^{i \alpha \phi(z)}:: e^{i \beta \phi(w)}:=(z-w)^{\alpha \beta}: e^{i \alpha \phi(z)+i \beta \phi(w)}:
$$

and therefore

$$
\psi(z) \psi^{*}(w)=\frac{w}{z-w}: e^{i \phi(z)-i \phi(w)}:
$$

Let us introduce the "Hamiltonians"

$$
\begin{aligned}
& H(T) \equiv \sum_{k=1}^{\infty} T_{k} J_{k} \\
& \bar{H}(\bar{T}) \equiv \sum_{k=1}^{\infty} \bar{T}_{k} \bar{J}_{k}
\end{aligned}
$$

Then one can show 28] that the following most important formulas hold:

$$
\begin{array}{r}
\langle n| \psi(z)=z^{n-1}\langle n-1| \exp \left[H\left(T-\epsilon\left(z^{-1}\right)\right)\right] \\
\equiv z^{n-1} \hat{X}(z, T)\langle n-1| e^{H(T)} \\
\langle n| \psi^{*}(z)=z^{-n}\langle n+1| \exp \left[H\left(T+\epsilon\left(z^{-1}\right)\right)\right] \\
\equiv z^{-n} \hat{X}^{*}(z, T)\langle n+1| e^{H(T)}
\end{array}
$$

\footnotetext{
${ }^{15}$ In this Appendix the normal ordering is taken with respect to the bosonic vacuum.
} 
where

$$
\begin{gathered}
\hat{X}(z, T)=e^{\xi(z, T)} e^{-\xi\left(z, \tilde{\partial}_{T}\right)} \\
\hat{X}^{*}(z, T)=e^{-\xi(z, T)} e^{\xi\left(z, \tilde{\partial}_{T}\right)}
\end{gathered}
$$

\section{A2. The derivation of the formula (37)}

In this Appendix as an example we demonstrate the derivation of formula (12) [42].

Since

$$
\begin{gathered}
\langle n-N| \psi^{*}\left(z_{1}\right) \ldots \psi^{*}\left(z_{N}\right)= \\
=(-1)^{N(N-1) / 2} \prod_{i=1}^{N} z_{i}^{1-n} \Delta(z)\langle n| \exp \left[H\left(\sum_{i=1}^{N} \epsilon\left(z_{i}^{-1}\right)\right)\right]
\end{gathered}
$$

and

$$
\begin{gathered}
\psi\left(w_{N}\right) \ldots \psi\left(w_{1}\right)|n-N\rangle= \\
=\prod_{i=1}^{N} w_{i}^{n-N} \Delta(w) \exp \left[H\left(\sum_{i=1}^{N} \epsilon\left(w_{i}\right)\right)\right]|n\rangle
\end{gathered}
$$

then, obviously,

$$
\begin{array}{r}
\left\langle n-N\left|\psi^{*}\left(z_{1}\right) \ldots \psi^{*}\left(z_{N}\right) \mathcal{G} \psi\left(w_{N}\right) \ldots \psi\left(w_{1}\right)\right| n-N\right\rangle= \\
=(-1)^{N(N-1) / 2} \Delta(z) \Delta(w) \prod_{i=1}^{N} z_{i}^{1-n} w_{i}^{N-n} \times \\
\times\left\langle n\left|\exp \left[H\left(\sum_{i=1}^{N} \epsilon\left(z_{i}^{-1}\right)\right)\right] \mathcal{G} \exp \left[H\left(\sum_{i=1}^{N} \epsilon\left(w_{i}\right)\right)\right]\right| n\right\rangle
\end{array}
$$

and, therefore, in the case of $N$ Miwa variables

$$
\begin{gathered}
\tau(T, \bar{T})=\frac{\langle n-N|\mathcal{G}| n-N\rangle}{\Delta\left(z^{-1}\right) \Delta(w)} \times \\
\times \operatorname{det} \frac{\left\langle n-N+1\left|e^{\sum \frac{1}{k} J_{k} z^{-k}} \mathcal{G} e^{\sum \frac{1}{k} \bar{J}_{k} w^{k}}\right| n-N+1\right\rangle}{\langle n-N|\mathcal{G}| n-N\rangle}
\end{gathered}
$$




\section{A3. Determinant representations for $\tau$-functions}

In this Appendix we derive the determinant representation of $\tau$-functions in terms of ordinary (not Miwa) times. Some relations used in the derivation are necessary to obtain formulas of the section 5.2. We follow the line of the paper [30].

Let $\mathcal{G}$ be an arbitrary element of the Clifford group which does not mixes the $\psi$ - and $\psi^{*}$ modes :

$$
\mathcal{G}=: \exp \left[\sum \mathcal{A}_{k m} \psi_{k} \psi_{m}^{*}\right]:
$$

where : : denotes throughout this Appendix the normal ordering with respect to the Dirac vacuum $|0\rangle$. Then it is well known 28, 43] that

$$
\tau_{n}(x, y)=\left\langle n\left|e^{H(x)} \mathcal{G} e^{-\bar{H}(y)}\right| n\right\rangle
$$

solves the two-dimensional Toda lattice hierarchy, i.e. is the solution to the whole set of the Hirota bilinear equations. Any particular solution depends only on the choice of the element $\mathcal{G}$ (or, equivalently it can be uniquely described by the matrix $\mathcal{A}_{k m}$ ). From eqs.(A1) one can conclude that any element in the form (A23) rotates the fermionic modes as follows

$$
\mathcal{G} \psi_{k} \mathcal{G}^{-1}=\psi_{j} R_{j k}, \mathcal{G} \psi_{k}^{*} \mathcal{G}^{-1}=\psi_{j}^{*} R_{k j}^{-1},
$$

where the matrix $R_{j k}$ can be expressed through $\mathcal{A}_{j k}$ (see 44]). We will see below that the general solution (A23) can be expressed in the determinant form with explicit dependence of $R_{j k}$. In order to calculate $\tau$-function we need some more notations. Using commutation relations (A1) one can obtain the evolution of $\psi(z)$ and $\psi^{*}(z)$ in times $\left\{x_{k}\right\},\left\{y_{k}\right\}$ in the form

$$
\begin{gathered}
\psi(z, x) \equiv e^{H(x)} \psi(z) e^{-H(x)}=e^{\xi(x, z)} \psi(z), \\
\psi^{*}(z, x) \equiv e^{H(x)} \psi^{*}(z) e^{-H(x)}=e^{-\xi(x, z)} \psi^{*}(z) ; \\
\psi(z, \bar{y}) \equiv e^{\bar{H}(y)} \psi(z) e^{-\bar{H}(y)}=e^{\xi\left(y, z^{-1}\right)} \psi(z),
\end{gathered}
$$




$$
\psi^{*}(z, \bar{y}) \equiv e^{\bar{H}(y)} \psi^{*}(z) e^{-\bar{H}(y)}=e^{-\xi\left(y, z^{-1}\right)} \psi(z)
$$

where

$$
\xi(x, z)=\sum_{k=1}^{\infty} x_{k} z^{k} .
$$

Using the definition of the Shur polynomials $P_{k}(x)$ (46), from eqs. (A25)-(A28) one can easily obtain the evolution of the fermionic modes:

$$
\begin{gathered}
\psi_{k}(x) \equiv e^{H(x)} \psi_{k} e^{-H(x)}=\sum_{m=0}^{\infty} \psi_{k-m} P_{m}(x), \\
\psi_{k}^{*}(x) \equiv e^{H(x)} \psi_{k}^{*} e^{H(x)}=\sum_{m=0}^{\infty} \psi_{k+m}^{*} P_{m}(-x) \\
\psi_{k}(\bar{y}) \equiv e^{\bar{H}(y)} \psi_{k} e^{-\bar{H}(y)}=\sum_{m=0}^{\infty} \psi_{k+m} P_{m}(y) ; \\
\psi_{k}^{*}(\bar{y}) \equiv e^{\bar{H}(y)} \psi_{k}^{*} e^{-\bar{H}(y)}=\sum_{m=0}^{\infty} \psi_{k-m}^{*} P_{m}(-y) .
\end{gathered}
$$

It is useful to introduce the totally occupied state $|-\infty\rangle$ which satisfies the requirements (c.f. with (A7))

$$
\psi_{i}^{*}|-\infty\rangle=0, i \in \mathbf{Z}
$$

Then any shifted vacuum can be generated from this state as follows:

$$
|n\rangle=\psi_{n-1} \psi_{n-2} \ldots|-\infty\rangle \text {. }
$$

Note that the action of an any element $\mathcal{G}$ of the Clifford group (and, as consequence, the action of $\left.e^{-\bar{H}(y)}\right)$ on $|-\infty\rangle$ is very simple: $\mathcal{G}|-\infty\rangle \sim|-\infty\rangle$, so using (A31) and (A32) one can obtain from eq. A23):

$$
\begin{aligned}
\tau_{n}(x, y)= & \left\langle-\infty\left|\ldots \psi_{n-2}^{*}(-x) \psi_{n-1}^{*}(-x) \mathcal{G} \psi_{n-1}(-\bar{y}) \psi_{n-2}(-\bar{y}) \ldots\right|-\infty\right\rangle \sim \\
& \left.\sim \operatorname{det}\left[\left\langle-\infty\left|\psi_{i}^{*}(-x) \mathcal{G} \psi_{j}(-\bar{y}) \mathcal{G}^{-1}\right|-\infty\right\rangle\right]\right|_{i, j \leq n-1} .
\end{aligned}
$$


Using (A24) it is easy to see that

$$
\mathcal{G} \psi_{j}(-\bar{y}) \mathcal{G}^{-1}=\sum_{m, k} P_{m}(-y) \psi_{k} R_{k, j+m}
$$

and the "explicit" solution of the two-dimensional Toda lattice has the determinant representation:

$$
\left.\tau_{n}(x, y) \sim \operatorname{det} \hat{H}_{i+n, j+n}(x, y)\right|_{i, j<0}
$$

where

$$
\hat{H}_{i j}(x, y)=\sum_{k, m} R_{k m} P_{k-i}(x) P_{m-j}(-y) .
$$

The ordinary solutions to KP hierarchy [28] correspond to the case when the whole evolution depends only of positive times $\left\{x_{k}\right\}$; negative times $\left\{y_{k}\right\}$ serve as parameters which parameterize the family of points in Grassmannian and can be absorbed by re-definition of the matrix $R_{k m}$. Then $\tau$-function of (modified) KP hierarchy has the form

$$
\tau_{n}(x)=\left.\left\langle n\left|e^{H(x)} \mathcal{G}(y)\right| n\right\rangle \sim \operatorname{det}\left[\sum_{k} R_{k, j+n}(y) P_{k-i-n}(x)\right]\right|_{i, j<0},
$$

where $\mathcal{G}(y) \equiv \mathcal{G} e^{-\bar{H}(y)}$ and

$$
R_{k j}(y) \equiv \sum_{m} R_{k m} P_{m-j}(-y)
$$

\section{Appendix B. Higher Casimirs}

In this Appendix we discuss some formulas on higher Casimirs which appear in the formulas of the section 6 .

We start with the definitions from the book [25]. For GL(N) the standard Casimirs $C_{n}$ have the form Eq.(50). Let, as before, $\alpha \equiv\left(m_{1}, \ldots, m_{N}\right)$ be the signature of irreducible representation and

$$
k_{i}=m_{i}+N-i
$$


Then eigenvalues of Casimirs are:

$$
c_{n}(\alpha)=\sum_{i=1}^{N} k_{i}^{n} \gamma_{i}
$$

where

$$
\gamma_{i}=\prod_{i \neq j}\left(1-\frac{1}{k_{i}-k_{j}}\right)
$$

This is the rather ugly expression. From this it is hard to see (but, nevertheless, it is true) that $c_{n}(\alpha)$ are symmetric polynomials of $\left\{k_{i}\right\}$ since generating function for eigenvalues

$$
c(\alpha, z) \equiv \sum_{n=0}^{\infty} c_{n}(\alpha) z^{n}=\sum_{i=1}^{N} \frac{\gamma_{i}}{1-k_{i} z}
$$

can be represented in the form

$$
c(\alpha, z)=\frac{1}{z}[1-\pi(\alpha, z)]
$$

where

$$
\pi(\alpha, z)=\prod_{i=1}^{N}\left(1-\frac{z}{1-k_{i} z}\right)
$$

From the last expression the symmetricity and polynomiality of $c_{n}(\alpha)$ w.r.t. $\left\{k_{i}\right\}$ is obvious.

Now let us use this way of [25] in order to derive very "simple" expression for eigenvalues. From (B6)

$$
\pi(\alpha, z)=\prod_{i=1}^{N} \frac{1-\left(k_{i}+1\right) z}{1-k_{i} z}=\exp \left\{\sum_{m=0}^{\infty} \frac{z^{m}}{m} \sum_{i=1}^{N}\left[k_{i}^{m}-\left(k_{i}+i\right)^{m}\right]\right\}=\sum_{n=1}^{\infty} z^{n} P_{n}(\tilde{T})
$$

where

$$
\tilde{T}_{n}=\frac{1}{n} \sum_{i}\left[k_{i}^{n}-\left(k_{i}+1\right)^{n}\right.
$$

and thus from (B5)

$$
c_{n}(\alpha)=-P_{n+1}(\tilde{T})
$$

Examples:

$$
\begin{gathered}
c_{0}(\alpha)=N \\
c_{1}(\alpha)=\sum_{i} k_{i}-\frac{1}{2} N(N-1)=\sum_{i} m_{i} \\
c_{2}(\alpha)=\sum_{i} k_{i}^{2}-(N-1) \sum_{i} k_{i}-\frac{1}{6} N(N-1)(N-2)=\sum_{i} m_{i}^{2}+\sum_{i<j}\left(m_{i}-m_{j}\right)
\end{gathered}
$$

etc. These expressions (as the Shur polynomials) have the very nice form in the exponential factor in the bilinear combination of characters. But, unfortunately, the standard Casimirs $c_{n}$ 
with $n>2$ are not factorizable in the simple product form. This is an obstacle to turn to the determinant form and, as the consequence, to conclude that our object is a $\tau$-function. Thus, we propose again to use new Casimirs like $\sum k_{i}^{n}$ in order to reproduce (103) (if we restrict ourself with " $c_{0}+c_{1}+c_{2}$ " - there is no difference between these two different definitions, of course). It is in complete analogy with using only terms of the type $\operatorname{tr} X^{n}$, but not $\operatorname{tr} X^{n} \operatorname{tr} X^{m}$ in GKM (2).

\section{References}

[1] M.Douglas Phys.Lett., 238B (1990) 176

[2] A.Gerasimov et al. Nucl.Phys., B357 (1991) 565

[3] S.Kharchev et al. Phys.Lett., 275B (1992) 311; Nucl.Phys., B380 (1992) 181

[4] A.Marshakov, Int.J.Mod.Phys., A8 (1993) 3831

[5] A.Morozov, Integrability and matrix models, Preprint ITEP-M2/93

[6] V.Kazakov, A.Migdal, Nucl.Phys., B397 (1993) 214

[7] C.Itzykson and J.-B.Zuber, J.Math.Phys., 21 (1980) 411

[8] S.Kharchev, A.Marshakov, On $p-q$ duality and explicit solutions in $c \leq 12 d$ gravity models, Preprint NORDITA-93/20, FIAN/TD/04-93

[9] A.Mironov, On GKM description of multi-criticality in 2d gravity, Preprint FIAN/TD$17 / 92$

[10] S.Kharchev et al., Mod.Phys.Lett., A8 (1993) 1047-1061

[11] A.Losev, Descendants constructed from matter field in topological Landau-Ginzburg theories coupled to topological gravity, Preprint ITEP, hep-th 9211090;

A.Losev, I.Polyubin, On Connection between Topological Landau-Ginzburg Gravity and Integrable Systems, Preprint UUITP-2/93, hep-th 9305079

[12] A. Migdal, Zh.Eksp.Teor.Fiz. 69 , 810 (1975) (Sov.Phys.JETP. 42 413) 
[13] B. Rusakov, Mod.Phys.Lett., A5, 693 (1990)

[14] D.Gross, W.Taylor, Twists and Wilson loops in the string theory of two dimensional QCD, Preprint CERN-TH.6827/93, PUPT-1382, LBL-33767, UCB-PTH-93/09

[15] A.Gorsky, N.Nekrasov, Hamiltonian systems of Calogero type and two dimensional YangMills theory, Preprint UUITP-6/93, ITEP-20/93

[16] J.Minahan, A.Polychronakos, Equivalence of Two Dimensional QCD and the c=1 Matrix Model, Preprint CERN-TH 6843/93, UVA-HET-93-02

[17] M.Douglas, Conformal Field Theory Techniques for Large N Group Theory, Preprint RU-93-13, NSF-ITP-93-39

M.Douglas, V.Kazakov, Large $N$ Phase Transition in Continuum $Q C D_{2}$, Preprint LPTENS-93/20, RU-93-17

[18] M.Caselle, A.D'Adda, L.Magnea and S.Panzeri, Two dimensional QCD is a one dimensional Kazakov-Migdal model, Preprint DFTT-15/93

[19] J.Distermaat, G.Heckmann, Inv.Math., 69 (1982) 259

M.Blau, E.Keski-Vakkuri, A.Niemi, Phys.Lett., B246 (1990) 92

E.Keski-Vakkuri, A.Niemi, G.Semenoff, O.Tirkkonen, Phys.Rev., D44 (1991) 3899

A.Hietamaki, A.Morozov, A.Niemi, K.Palo, Phys.Lett., B263 (1991) 417

A.Morozov, A.Niemi, K.Palo, Phys.Lett., B271 (1991) 365; Nucl.Phys., B377 (1992) 295

[20] I.Kogan, A.Morozov, G.Semenoff, N.Weiss, Nucl.Phys., B395 (1993) 547

[21] A.Migdal, Mod.Phys.Lett., A8 (1993) 359

[22] A.Alekseev, L.Faddeev, S.Shatashvili, J.Geom.Phys., v.3 (1989)

[23] A.Morozov, Mod. Phys. Lett. A7 (1992) 3503

[24] S.Shatashvili, Comm. Math. Phys. 154 (1993) 421

[25] D.P.Zhelobenko, Compact Lie group and their representations, Nauka, Moscow, 1977 
[26] E. Witten, Comm. Math. Phys., 141, 153 (1991)

[27] G.Moore, N.Seiberg, Comm.Math.Phys., 123 (1989) 177

[28] M.Sato, Y.Sato, Soliton equations as dynamical systems in an infinite-dimensional Grassmannian, in: Nonlinear partial differential equations in applied sciences, P.Lax, H.Fujita, G.Strang (North-Holland, Amsterdam, 1982)

E.Date, M.Jimbo, M.Kashiwara, T.Miwa, Transformation groups for soliton equations, RIMS Symp. "Non-linear integrable systems - classical theory and quantum theory" (World scientific, Singapore, 1983)

[29] S.Kharchev et al. Nucl.Phys., B366 (1991) 569

[30] S.Kharchev et al. Nucl.Phys., B397 (1993) 379

[31] D.E.Littlewood, The theory of group characters and matrix representations of groups, Oxford, 1958

[32] G.Segal, G.Wilson, Publ.Math. I.H.E.S., 61 (1985) 1

[33] See, e.g. Y.Ohta, J.Satsuma, D.Takahashi and T.Tokihiro, Prog.Theor.Phys.Suppl., 94 (1988) 210

[34] V.Kac, Infinite-dimensional Lie algebras, Cambridge University Press, Cambridge, 1985

[35] A.Marshakov, On string field theory for $c \leq 1$, Preprint FIAN/TD/08-92

[36] R.Dijkgraaf, G.Moore, R.Plesser, Nucl.Phys., B394 (1993) 356

[37] Harish-Chandra, Amer.J.Math., 80 (1958) 241

[38] Olshanetsky, Perelomov, Phys.Rept., 94 (1983) 313

[39] F.Berezin, F.Karpelevich, Dokl.Akad.Nauk SSSR, 118 (1958) 9-12

[40] A.Niemi, O.Tirkkonen, On Exact Evaluation of Path Integrals, Preprint HU-TFT-93-7, UU-ITP $3 / 93$

[41] A.Marshakov, A.Mironov, A.Morozov, Phys.Lett. 274B (1992) 280 
[42] S.Kharchev, A.Mironov, $\tau$-function of two-dimensional Toda lattice and quantum deformations of the integrable hierarchies, Preprint FIAN/TD-02/93

[43] K.Ueno, K.Takasaki, Adv.Studies in Pure Math., 4 (1984) 1

[44] M.Jimbo, T.Miwa, M.Sato, Holonomic quantum fields, $I-V$ : Publ.RIMS, Kyoto Univ., 14 (1978) 223; 15 (1979) 201, 577, 871, 531 